\title{
Recent Advances in Single Crystal Diamond Device Fabrication for Photonics, Sensing and Nanomechanics
}

\author{
Dipti Rani, Oliver Roman Opaluch and Elke Neu* \\ Fachbereich Physik, Technische Universität Kaiserslautern, Erwin-Schrödinger-Strasse, D-67663 Kaiserslautern, \\ Germany; Diptidahiya@gmail.com (D.R.); opaluch@rhrk.uni-kl.de (O.R.O.) \\ * Correspondence: nruffing@rhrk.uni-kl.de
}

\begin{abstract}
In the last two decades, the use of diamond as a material for applications in nanophotonics, optomechanics, quantum information, and sensors tremendously increased due to its outstanding mechanical properties, wide optical transparency, and biocompatibility. This has been possible owing to advances in methods for growth of high-quality single crystal diamond (SCD), nanofabrication methods and controlled incorporation of optically active point defects (e.g., nitrogen vacancy centers) in SCD. This paper reviews the recent advances in SCD nano-structuring methods for realization of micro- and nano-structures. Novel fabrication methods are discussed and the different nanostructures realized for a wide range of applications are summarized. Moreover, the methods for color center incorporation in SCD and surface treatment methods to enhance their properties are described. Challenges in the upscaling of SCD nano-structure fabrication, their commercial applications and future prospects are discussed.
\end{abstract}

Keywords: diamond; nanofabrication; photonics; quantum technologies

Citation: Rani, D.; Opaluch, O.R.;

Neu, E. Recent Advances in Single Crystal Diamond Device Fabrication for Photonics, Sensing and Nanomechanics. Micromachines 2021, 12, 36. https://doi.org/ $10.3390 / \mathrm{mi1} 2010036$

Received: 14 December 2020 Accepted: 29 December 2020 Published: 30 December 2020

Publisher's Note: MDPI stays neutral with regard to jurisdictional clai$\mathrm{ms}$ in published maps and institutional affiliations.

Copyright: $(\odot 2020$ by the authors. Licensee MDPI, Basel, Switzerland. This article is an open access article distributed under the terms and conditions of the Creative Commons Attribution (CC BY) license (https:// creativecommons.org/licenses/by/ $4.0 /)$.

\section{Introduction}

Diamond is an outstanding material for micro/nanofabrication of optomechanical, photonic and sensor components owing to its exceptional mechanical properties, broad optical transparency combined with biocompatibility in both bulk and nano-structures. High quality, high purity single crystal diamond (SCD) is mainly grown via chemical vapor deposition (CVD) methods. SCD is mostly limited to sizes below $5 \mathrm{~mm} \times 5 \mathrm{~mm}$ and a costly material. Only recently, SCD has become available in sizes up to $100 \mathrm{~mm}$ wafers via heteroepitaxy. Consequently, diamond based optomechanical and nanomechanical components are realized often on polycrystalline diamond films which can be easily grown on wafer scale. However, using polycrystalline material will limit the performance of photonic components and sensors due to light scattering, strain and defects [1]. With advancement in nanofabrication methods, various diamond structures have been realized including nanomechanical, nanophotonic and optomechanical systems. Nanomechanical systems, including nanoresonators, allow ultrahigh sensitivities in force detection and realizing optomechanical systems which couple optical and mechanical modes [2,3]. On the other hand, photonic components including nanopillar waveguides, photonic crystals $(\mathrm{PhC})$, optical antennas, metalenses, solid immersion lenses (SIL) and other optical resonators are used for light manipulation, propagation, and trapping. Optically active point defects called color centers in diamond have proven useful for a wide range of applications which span from sensing magnetic fields e.g., in nano magnetic resonance, single molecule detection and single photon emission to optomechanics, etc. [3-5]. The most explored point defect in diamond, the nitrogen vacancy $(\mathrm{NV})$ center provides an optically readable electron spin at room temperature even for individual color centers. However, photon collection efficiency from NV centers in bulk diamond is intrinsically limited due to the large refractive index of diamond $(\mathrm{n} \approx 2.4)$ and total internal reflection at the diamond-air interface. Exceptional efforts were made to engineer high quality NV centers with long 
spin coherence times and realize diamond structures for efficient light extraction. Realizing such structures, often embedding single NV centers, requires growth and nanofabrication of high quality SCD. In recent years, many novel nano-structures have been realized for advanced sensing applications with NV centers replacing micro/nano devices developed earlier. However, NV center based devices are not perfectly suited for many quantum communication applications, as e.g., quantum repeaters, since their photon throughput is limited due to long fluorescence lifetime $(\approx 11 \mathrm{~ns})$ and weak emission into zero phonon line (ZPL, $4 \%$ at room temperature) [6]. Moreover, NV centers have a permanent dipole moment increasing their susceptibility to surrounding electrostatic fields and decreasing the rate of generation of indistinguishable photons [7].

Consequently, different types of color centers with emission ranging from visible to infrared are currently investigated. Among those, group-IV atoms based color centers such as silicon-vacancy $(\mathrm{SiV})$, germanium-vacancy $(\mathrm{GeV})$, tin-vacancy $(\mathrm{SnV})$, and lead-vacancy $(\mathrm{PbV})$ are worth mentioning which show narrow emission and promising coherence properties at low temperatures [6]. They possess inversion symmetry and thus are less susceptible to variations in electrostatic fields. The $\mathrm{GeV}$ and $\mathrm{SnV}$ centers are advantageous due to their larger energy splitting in the ground state which results in better coherent spin manipulation. $\mathrm{GeV}$ centers yield higher quantum efficiency (in comparison to $\mathrm{SiV}$ centers) and are thus favorable for applications in quantum optics and nanophotonics [7]. All these color centers show promising emission properties, novel wavelengths and have opened wide directions in scientific exploration. Extensive discussion about the creation and applications of color centers is beyond the scope of this review.

Diamond nano-structures are developed using top-down and bottom-up approaches. Top-down approaches include SCD structuring from high quality bulk substrates $\left(\approx 5 \times 5 \times(0.02-0.05) \mathrm{mm}^{3}\right)$ using state of the art lithography techniques and plasma etching methods. Impurity atoms forming color centers are introduced into SCD before or after nano-structuring often at precise locations via ion implantation. Within bottom-up approaches, diamond nano-structures are formed during diamond growth using CVD processes [8-10]. Defects are introduced via modifying the growth process (e.g., introduction of nitrogen) during CVD synthesis [3,11]. Bottom-up synthesis yields diamond nano-structures without the danger of inducing plasma etching induced defects. However, the density of color centers and shape/configuration of nano-structures is difficult to control via these methods. On the other hand, top-down approaches allow to create nano-structures with well-defined characteristics (shape, defect density) with high precision, but, require advanced nanofabrication and plasma etching which enhances processing costs $[4,12]$.

In this review, we mainly focus on the advancement in top-down fabrication methods in the last 3 years for structuring of bulk diamond into various nano-structures, which has not been done recently. Complementary to the previous reviews by Schroeder et al. [13] and Aharonovich et al. [14], which reported mainly the fabrication of diamond photonic devices, we describe the different micro/nano-structures that were realized and are suitable for applications in quantum photonics, sensing, mechanics, optomechanics, etc. The surface modification methods developed for improvement of device properties are discussed. We also discuss challenges hindering mass production of such devices and possible solutions.

This review is organized as follows: Section 1 overviews the nano-structuring of diamond including incorporation of different color centers; Section 2 introduces the nanophotonic components and the methods deployed to realize different nanophotonic structures; Section 3 introduces the nanomechanical components and describes the different structures realized; Sections 4 and 5 describe the developed optomechanical structures and other nano-structures, respectively; Section 6 summarizes the review and gives an outlook on how to improve diamond nano-structuring methods to upscale production and enhance reliability. 


\section{Why Nano-Structuring of Diamond}

Several commercial suppliers (e.g., Element 6 [15], Applied Diamond Inc. [16], AuDiaTec [17],) offer bulk diamond substrates of different size, quality, orientation, and defect density. Typically, substrates are characterized for their surface roughness and polishing wedge prior to nano-structuring. Structuring of diamond requires patterning of the resist mask using lithography techniques based on electron-beam (EBL), ultra-violet (UVL), nanoimprint (NIL) or laser or two photon lithography [18-22]. Owing to the high inertness of diamond, wet-etching methods are not suitable and thus transferring the pattern to the diamond is carried out using reactive ion etching (RIE). For etching of diamond, plasmas of special gases (oxygen/argon or chlorine-based mixtures) at high powers mostly in inductively coupled reactive ion etching (ICP-RIE) are used. Specific resists such as PMMA on hard masks layers $\left(\mathrm{Al}_{2} \mathrm{O}_{3}, \mathrm{Al}, \mathrm{W}\right)$ or hydrogen silsesquioxane (HSQ) are typical material systems.

Over the last decade, various nano-structures (nanopillars, nanowires, beams, nanogratings, etc.) were realized with $\mathrm{NV}$ centers incorporated via implantation of nitrogen atoms in high purity SCD. Although ion implantation precisely introduces dopants with optimal density and depth, it induces surface and lattice damage which affects the properties of the final devices. To overcome the limitation of ion implantation, an alternative approach includes high energy electron irradiation on ultrapure diamond to transform native nitrogen impurities into NV centers [23]. Nano-structures realized through such approaches are suitable for applications in quantum information processing, magnetometry, microscopy, nano magnetic resonance imaging, etc. $[1,2,5]$.

\section{Photonic Components}

\subsection{Basics of Photonic Components}

Nanophotonic platforms are capable of interfacing optical elements (e.g., waveguides, lenses) with solid-state quantum emitters (e.g., luminescent color centers) and are widely explored in quantum technologies. In diamond based components for quantum information, photons interconnect spins associated with individual luminescent color centers (e.g., NV centers) thereby enabling entanglement between distant spins [24]. Quantum information is stored in the electron spins of NVs or nuclear spins of nearby atoms in the diamond whereas coherent state manipulation is achieved via applying microwave or RF fields. The information is communicated via encoding the spin information into degrees of freedom of the photons. However, only photons from the purely electronic transition namely the zero phonon line (ZPL) are usable in this context. Unfortunately, the emission spectrum of NV centers consists of ZPL and a broad phonon sideband with only $4 \%$ of emission into the ZPL. To boost usability of NV centers in quantum information, it is important to enhance emission into the ZPL using optical cavities [1,25]. In contrast, the non-resonant spin read-out used in room temperature sensing applications works also via the photons in the sideband and thus mostly concentrates on waveguide structures that allow for broadband collection enhancement.

The main figure of merit for optical cavities is the Purcell factor $\left(P_{F}\right)$ : It describes the enhancement in spontaneous emission rate for an optimally aligned dipole localized in the maximum of the cavity field:

$$
P_{F}=\frac{3}{4 \pi^{2}}\left(\frac{\lambda}{n}\right)^{3} \frac{Q}{V}
$$

where $V, n$ and $\lambda$ are the cavity mode volume, mode index and emission wavelength, respectively. The quality factor $Q$ describes the storage time of photons in the cavity and depends upon the electric field decay rate in the cavity $\kappa$ and the resonant frequency of the cavity $\omega_{c}$.

$$
Q=\frac{\omega_{c}}{2 \kappa}
$$

Thus, high quality factors $(Q)$ and/or a low mode volume $(V)$, resulting in long interaction times between the emitter and the photons and a strong electric field, are preferred. 
$Q$-factor and mode volume are used to characterize narrow bandwidth devices such as ring or disk resonators or photonic crystal resonators. In contrast, waveguide devices like nanopillars, lenses, gratings etc. will rather be characterized by their enhancement of the collection efficiency.

Most photonic structures are not suitable for randomly distributed centers as a defined placement within the nano-structure is mandatory for optimal coupling [26]. Large Qs are easily obtained in near-infrared regime in large resonators such as ring-resonators and in devices with large cavities which can be easily fabricated. For photonic crystal cavities, 1-D cavities result in high $Q$-factors and $P_{F}$ owing to the small mode volumes, in comparison to 2-D photonic cavities with same parameters. The combination of optimal nanofabrication and precise positioning of color centers remains challenging as detailed below.

\subsection{Fabrication of Thin SCD Membranes for Photonic Structures}

Realization of photonic components often requires high quality, SCD membranes/ structures of around few hundreds of nanometers which are difficult to grow in a controlled manner via bottom-up methods. Harnessing advanced nano-structuring methods for SCD, bulk SCD is structured into free standing, thin films $(\approx 200 \mathrm{~nm}$ thickness) or free standing nano-structures. The deployed fabrication methods are briefly described in the following paragraphs:

- The lift-off method (illustrated schematically in Figure 1A) where SCD is implanted with ions to create a sacrificial layer below the SCD surface. The layer is graphitized in an annealing process (ii) and removed via electrochemical etching (iii). The thin, peeled-off SCD membrane is transferred to another substrate (iv) and pristine quality SCD is overgrown while the original, ion-damaged SCD membrane is etched away [27].

- The thin down technique in which commercially available, high-purity $\operatorname{SCD}(\approx 50 \mu \mathrm{m}$ thick) is etched down to required thickness of few hundreds of nanometers via dry etching methods [28]. An example is illustrated in Figure 1B, thin SCD $(\approx 30 \mu \mathrm{m})$ is transferred to $\mathrm{SiO}_{2} / \mathrm{Si}$ substrates for easy handling (i) and dry etched (ii) to obtain thin SCD membranes $(\approx 200 \mathrm{~nm})$. For nano-structuring, the diamond $/ \mathrm{SiO}_{2} / \mathrm{Si}$ samples are spin coated (iii) and patterned via EBL (iv). The resist pattern is transferred into SCD using anisotropic dry etching processes (v), followed by resist mask removal.

- Direct cleavage method using a focused beam of ions such as $\mathrm{Ga}$ or $\mathrm{O}_{2}$ to directly cut (mill) SCD without any mask preparation. This method is limited in terms of scalability and the damage introduced due to implanted ions $[29,30]$.

- Angle etching method which transfers a pattern defined using EBL into SCD using two dry etching steps as shown in Figure 1C [31-33]. The lithographically defined resist pattern $(\mathrm{i}, \mathrm{ii})$ is first transferred into SCD via anisotropic etching (ICP-RIE), etching few hundreds of nanometers of SCD (iii). This is followed by dry etching at an oblique angle using ion beam etching (IBE) or by using a Faraday cage inside the RIE chamber to undercut the structures (iv). Finally, the resist mask is removed (v).

- The isotropic under-etching method in which a combination of anisotropic and isotropic oxygen etching (at elevated temperature) creates free standing nano-structures [34-36]. A hard mask pattern ( $\mathrm{SiN})$ is transferred into SCD (i). A conformal coating with $\mathrm{Al}_{2} \mathrm{O}_{3}$ is carried out using atomic layer deposition (ii). Subsequently, the top surface of $\mathrm{Al}_{2} \mathrm{O}_{3}$ is dry etched (iii) to keep only the sides of structure covered. A quasi-isotropic oxygen etching at $200^{\circ} \mathrm{C}$ is carried out to undercut the structure (iv). Finally, the mask is removed (v).

- Si membrane hard mask transfer method where re-usable Si membranes are used as hard masks, which are patterned using mature silicon microfabrication techniques. These membranes are transferred onto diamond substrates for creating free-standing membranes via dry etching methods [37].

Heteroepitaxially grown SCD might provide an alternative route to free-standing structures, however, it is still challenging to perform high quality SCD heteroepitaxy and 
high-quality layers typically have millimeter thickness. Recently, Schreck et al. [3,38] reported heteroepitaxial growth of a $100 \mathrm{~mm}$ diameter, (100)-oriented, millimeter thick, SCD wafer on Ir/YSZ (yttria stabilized zirconia)/Si substrate via CVD. Consequently, this material system might allow up-scaling to wafer scale processing, whereas the challenge of thin membrane fabrication and free-standing structures remains.

A
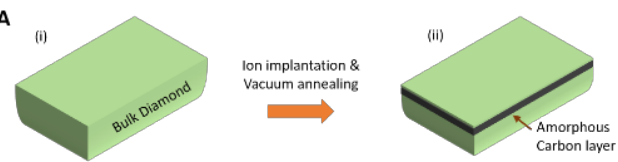
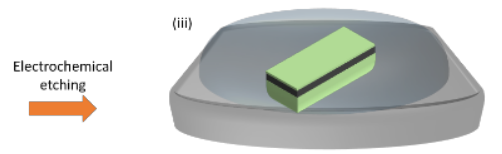
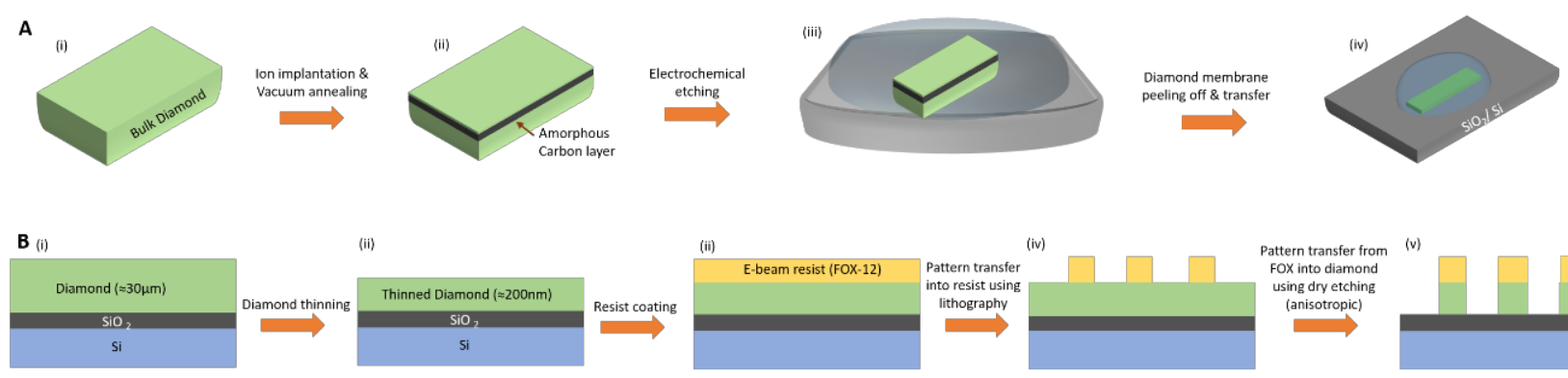

$C_{(i)}$
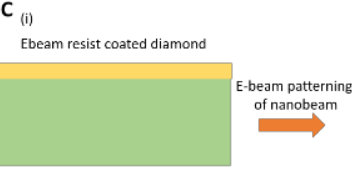

(ii)
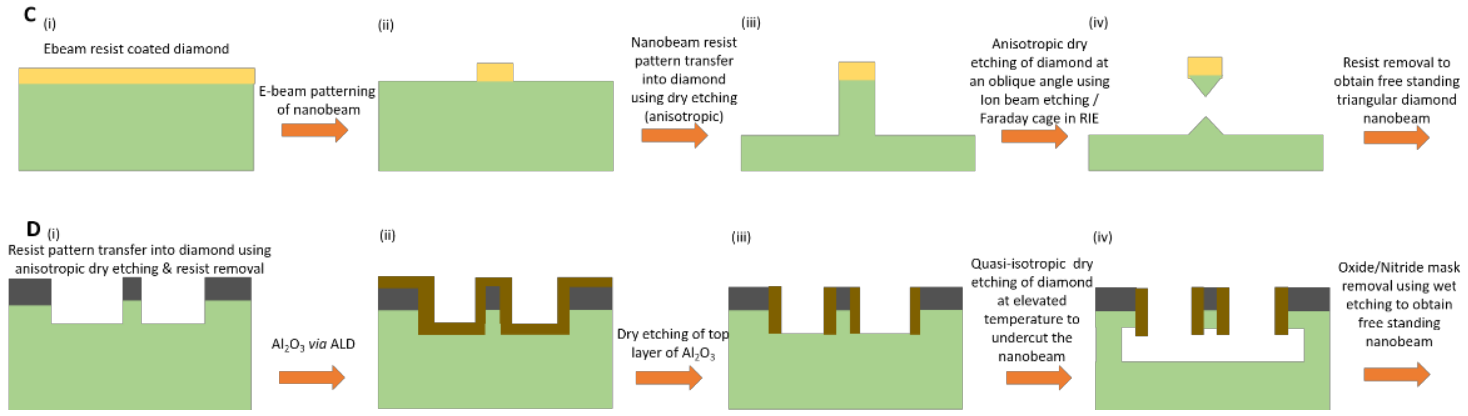

(iv)

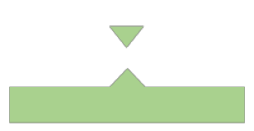

Figure 1. Schematics of different methods realized for diamond nanofabrication: (A) Lift-off method in which ions are implanted into bulk diamond to create an amorphous carbon layer (ii) few microns below the surface, followed by electrochemical etching of the damaged layer (iii) and transfer of the peeled-off diamond membrane over another substrate. Figures adapted from Ref. [27] originally published by The Royal Society of Chemistry under creative commons license CC BY 3.0; (B) Thin-down technique, where bulk diamond is transferred over another substrate (e.g., $\mathrm{SiO}_{2} / \mathrm{Si}$ ) and dry etched to obtain thin diamond membranes of $\approx 200 \mathrm{~nm}$ (i,ii). The structures in nanomembrane are created by patterning of the spin coated resist using E-beam lithography (iii, iv), and subsequent transfer into diamond using dry etching process (v). Figures adapted from Ref. [28] copyright IOP publishing, Reproduced with permission. All rights reserved; (C) Angled etching method in which lithographically defined nanobeam resist pattern (i,ii) is transferred over the underlying diamond substrate using anisotropic dry etching process (iii), followed by dry etching at an oblique angle using ion beam etching or Faraday cage in RIE (iv) to release the diamond nano-structures and subsequent resist mask removal to obtain free-standing, triangular nanobeam (v). Figures reprinted (adapted) with permission from [33,39] Copyright (2019) by the American Physical Society for Ref. [33], for Ref. [39] reprinted under creative commons license CC BY 4.0; (D) Isotropic undercut etching method, where a combination of anisotropic and isotropic dry etching processes is used to obtain free-standing diamond nano-structures. Nanobeam pattern transfer using hard mask into diamond via a combination of e-beam lithography and anisotropic etching (i). The patterned diamond substrate is coated conformally with $\mathrm{Al}_{2} \mathrm{O}_{3}$ via ALD technique (ii) and dry etched (iii) to remove the top layer of $\mathrm{Al}_{2} \mathrm{O}_{3}$. Subsequently quasi-isotropic oxygen etching is carried out to undercut the nanobeam structure (iv) and wet etching of hard mask to obtain free-standing nanobeam structure over bulk diamond (v). Figures reprinted (adapted) from [34,36], for Ref. [34] with the permission of AIP Publishing; for Ref [36] under creative commons license CC BY 4.0.

\subsection{High Q-Photonic Resonators in SCD}

\subsubsection{Photonic Crystal Resonator}

For quantum information, 1-dimensional nanobeams ( $\approx 200 \mathrm{~nm}$ thick) or 2-dimensional photonic crystal slabs with high-quality factors are advantageous. They potentially allow for large scale systems with multiple emitters per cavity coupled strongly in addition to arrays of multiple emitter-cavity nodes, photonic/spin quantum gates and quantum memories [1]. Such structures are mainly fabricated using two methods: SCD membranes 
thinned from bulk, and suspended SCD structures formed via angled etching [34]. For the first approach, obtained Q-factors are moderate: few thousand near the ZPL (e.g., NV ZPL). Furthermore, the yield of cavities across thinned membranes is often low due to the initial variations in thickness of the starting membranes, and thus presents a major challenge in scaling up of the fabrication. In a recent report, Mouradian et al., demonstrated quasi-isotropic oxygen under-cut etching to fabricate rectangular nanobeam cavities in SCD [34]. Due to the crystal plane dependent quasi-isotropic etching process, structures were pre-aligned with the fastest etching plane (100) to achieve rectangular cross-section of the nanobeams. The side view of the rectangular nanobeam photonic crystal cavities is illustrated in Figure 2A [34]. Rectangular nanobeam cavities have low out-of-plane scattering losses in contrast to the nanobeam cavities with triangular cross-sections. The developed fabrication process was extended to realize suspended 2-D photonic crystal $(\mathrm{PhC})$ nanocavity slabs (see Figure 2B) [35]. 2-D PhC slab cavities may improve $P_{F}$ in comparison to 1-D PhC, as they inhibit unwanted spontaneous emission out of the cavity in the 2-D bandgap. The developed quasi-isotropic etching process is highly reproducible, with minimum process optimization requirements and enables high Qs exceeding 14,000 within $1 \mathrm{~nm}$ of $\mathrm{NV}^{-} \mathrm{ZPL}(\lambda=637 \mathrm{~nm})$ as well as uniform cavity fabrication over an entire chip. However, controlling surface roughness of the isotropically etched structures is limited. Ref. [33] reports angled-etching of patterned diamond structures using ion beam etching instead of Faraday cage based ICP-RIE etching to overcome the limitations of non-uniform etch rates (due to the imperfections in Faraday cage mesh). These nanophotonic cavities were incorporated with $\mathrm{SiV}$ centers using lithographically masked implantation process and coupled with microwave coplanar waveguides ( $250 \mathrm{~nm}$ thick gold) onto the same chip to form an integrated quantum nanophotonic register.

\subsubsection{Other Resonators}

Other resonators such as ring resonators can be etched into suspended SCD membranes or structured into diamond-on-insulator. These structures are usually coupled with waveguides and are suitable for on-chip photonics. Microdisk resonators are generally stand-alone devices in which the spontaneous emission is out-coupled using evanescent fiber coupling and sustain 'whispering gallery' electromagnetic modes [1,7,43,44].

Recent reports $[7,44]$ realized SCD membranes $(\approx 300 \mathrm{~nm})$ with $\mathrm{GeV}$ color centers using a combination of lift-off method and CVD. Metallic (Ge) and germanium oxide $\left(\mathrm{GeO}_{2}\right)$ sources introduced uniformly distributed $\mathrm{GeV}$ centers during CVD growth and the damaged layer was etched-off using ICP-RIE. Photonic resonators (with $Q \approx 1500$ ) such as microdisks and microrings were fabricated using a combination of EBL and ICP-RIE [7]. In another approach, high- $\mathrm{Q} / \mathrm{V}$ microdisk device in SCD was realized using a combination of anisotropic and quasi-isotropic oxygen undercut etching method by Khanaliloo et al. [45].

A novel method called reactive ion beam angled etching (RIBAE) was recently developed for highly uniform and scalable processing of free-standing photonic and mechanical nano-structures [40]. RIBAE is derived from ion beam etching (IBE) or ion beam milling in which inert gases (such as Ar, Xe) used for sputtering of the target material are replaced by reactive gases such as chlorine or oxygen to extend the range of etchable materials. A race-track resonator realized in bulk SCD via RIBAE method is shown in Figure 2C. To achieve 3-D nano-structures using this method, an etch mask is patterned initially using conventional lithography techniques, followed by RIE with the sample mounted perpendicularly to the ion beam on a rotating sample stage. Subsequent etching is carried out by tilting the sample with respect to the ion beam resulting in uniform etching of the structures underneath the etch mask. The developed method yields highly uniform structures over large areas $\approx 200 \mathrm{~mm}$ in diameter and depends mainly on the size of the ion beam source [46]. 

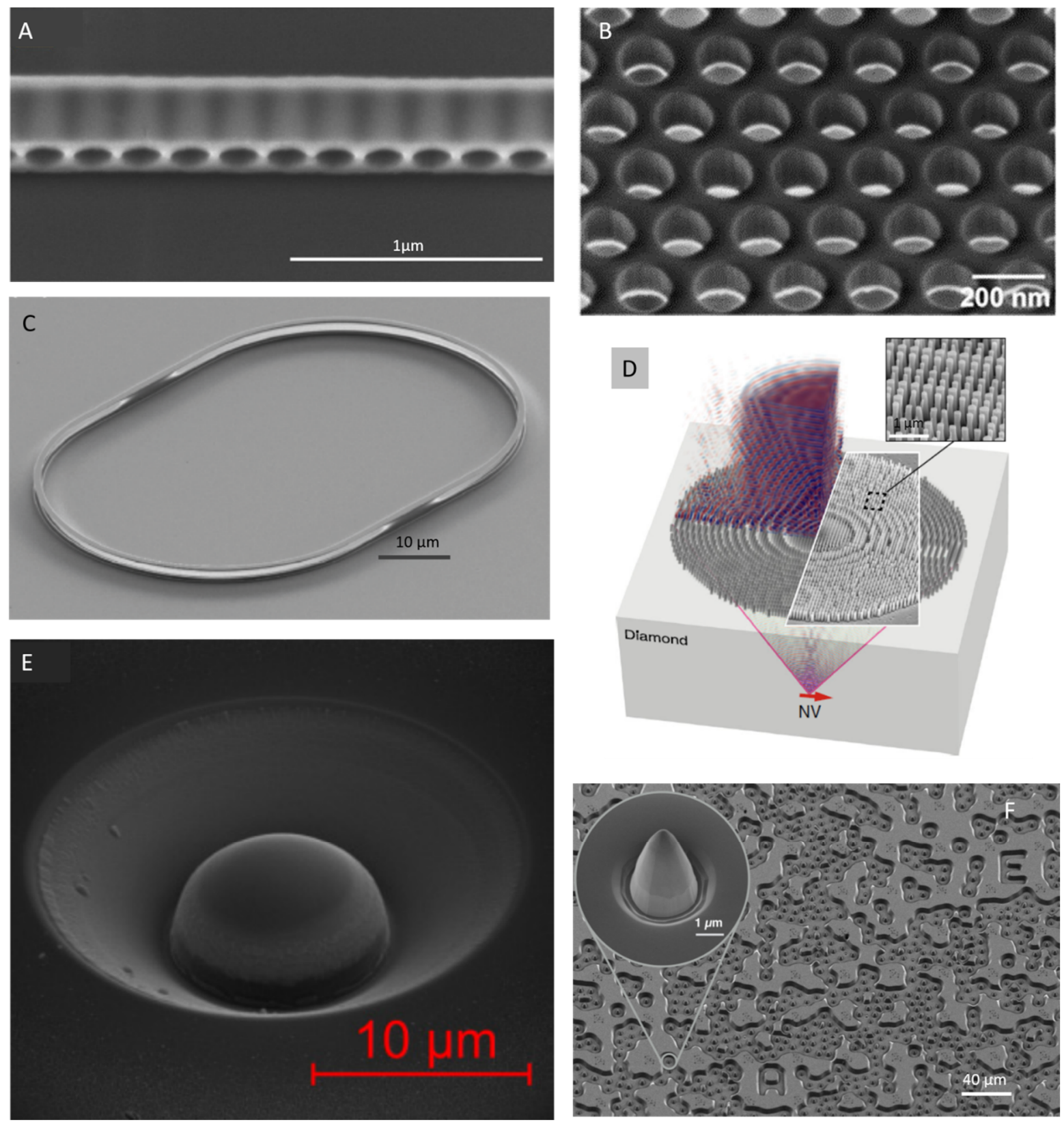

Figure 2. Scanning electron microscopy images (A-C) of different photonic components realized using lithography and dry etching processes: (A) rectangular nanobeam photonic crystal cavities (side view). Reprinted from Ref. [34], with the permission of AIP Publishing. (B) 2-D photonic crystals suspended in air, fabricated via quasi-isotropic undercut etching of diamond using oxygen plasma. Reprinted from Ref. [35], with the permission of AIP Publishing. (C) Diamond race track resonator fabricated via reactive ion beam angled etching. Image reprinted under creative commons CC-BY 4.0 from Ref. [40]. (D) Metalens consisting of a metasurface in which subwavelength pillars are etched into single crystal diamond substrate with naturally abundant NVs, using a combination of EBL and ICP-RIE. SEM image shown in the inset gives the zoomed-in view of the nanopillars forming the metalens. Figures reprinted from Ref. [41] under creative commons license CC BY 4.0. (E) SEM image of a solid immersion lens fabricated via focussed ion beam milling into single crystal HPHT diamond. In this example, the SIL incorporates native $\mathrm{GeV}$ center in the material. Figures reprinted from Ref. [30] under creative commons license CC BY 4.0. (F) Patterned diamond chip consisting of parabolic reflectors, individual one shown in the inset. Reflectors are $5 \mu \mathrm{m}$ in height, and are irregularly arranged on the diamond chip due to NV center location dependent patterning of resist mask. Reprinted (adapted) with permission from Ref. [42]. Copyright (2018) American Chemical Society. 


\subsection{Waveguides for Collection Enhancement}

After discussing resonator like structures, we now turn to the waveguide structures used to modify the emission properties of color centers.

\subsubsection{Metalens}

Metalenses are a comparably recent approach to collimate the emission from individual color centers: a metasurface composed of diamond nanopillars arranged in a regular pattern acts as an immersion lens and allows efficient coupling of color center emission to optical fibers. The diamond nanopillars constituting the metasurface are generally structured using a combination of EBL and RIE on a SCD surface [41,47]. A metasurface with subwavelength nanopillars etched into bulk SCD is shown in Figure 2D. Inset illustrates SEM image of the nanopillars [41].

\subsubsection{Solid Immersion Lenses (SIL)}

Solid immersion lenses (SIL) consist of a hemisphere into which the color center is embedded and overcome total internal reflection. However, photon collection remains limited due to Fresnel reflection [47] which can be minimized using anti-reflective coatings. These structures allow significant improvement in photon collection efficiency in comparison to color centers in bulk diamond. Moreover, SILs do not require to position color centers close to SCD surfaces, which might limit their spin and optical properties.

SILs are fabricated via RIE or focussed ion beam milling of hemispheres in high quality SCD. Especially for the later method, the SILs are annealed and acid cleaned to minimize crystal damage and contamination induced due to the milling process [29]. Figure 2E, shows an SEM image of a SIL fabricated via FIB milling [30].

\subsubsection{Parabolic Reflector}

Monolithic, parabolic reflectors for broadband photon extraction from color centers were recently reported by Wan et al. [20,42]. SEM image of the patterned diamond realized using conventional EBL and RIE is shown in Figure 2F, with individual parabolic reflector shown in the inset. The reflectors are irregularly arranged on the diamond chip as the mask has been aligned with respect to individual, predefined NV centers. These parabolic reflectors represent the brightest single-photon source in diamond.

\subsubsection{Single Crystal Diamond Nanopillars/Nanowires}

Diamond nanopillars and nanowires enhance the photon collection from color centers thus, they can be advantageous for single photon sources but also as monolithic diamond scanning probes in magnetometry. Various realizations of this geometry incorporating several types of color centers have been reported.

The first developed devices were SCD nanowires ( $200 \mathrm{~nm}$ wide and $2 \mu \mathrm{m}$ tall) fabricated via a combination of EBL and RIE on HPHT, (100)-oriented type Ib diamond. The nanowire acts as waveguide, enhancing the collection efficiency of the photons emitted from single NV centers [48]. Nanowire/nanopillar fabrication is scalable as large arrays of pillars can be etched and color centers can be precisely positioned in the pillars. (100)-oriented SCD is mostly employed for device fabrication owing to low-cost and easy polishing. However, (100)-oriented nanopillars have limitations [1]: For maximum photon collection efficiencies, the NV's dipoles should be perpendicular to the nanopillar axis. However, in (100)-oriented nanopillars, the NV's dipoles form an oblique angle with the pillar axis due to the aligning of NV center orientation with one of the four equivalent $<111>$ crystal directions. Recent availability of high-purity, high-quality (111)-oriented CVD grown SCD opened the possibility to fabricate (111) oriented pillars [49]. In this material, optimal alignment of NVs is reached as the NV's preferential alignment along the growth direction can be harnessed [50]. Some examples of nanopillars/nanowires realized are illustrated in Figure 3A-D. 


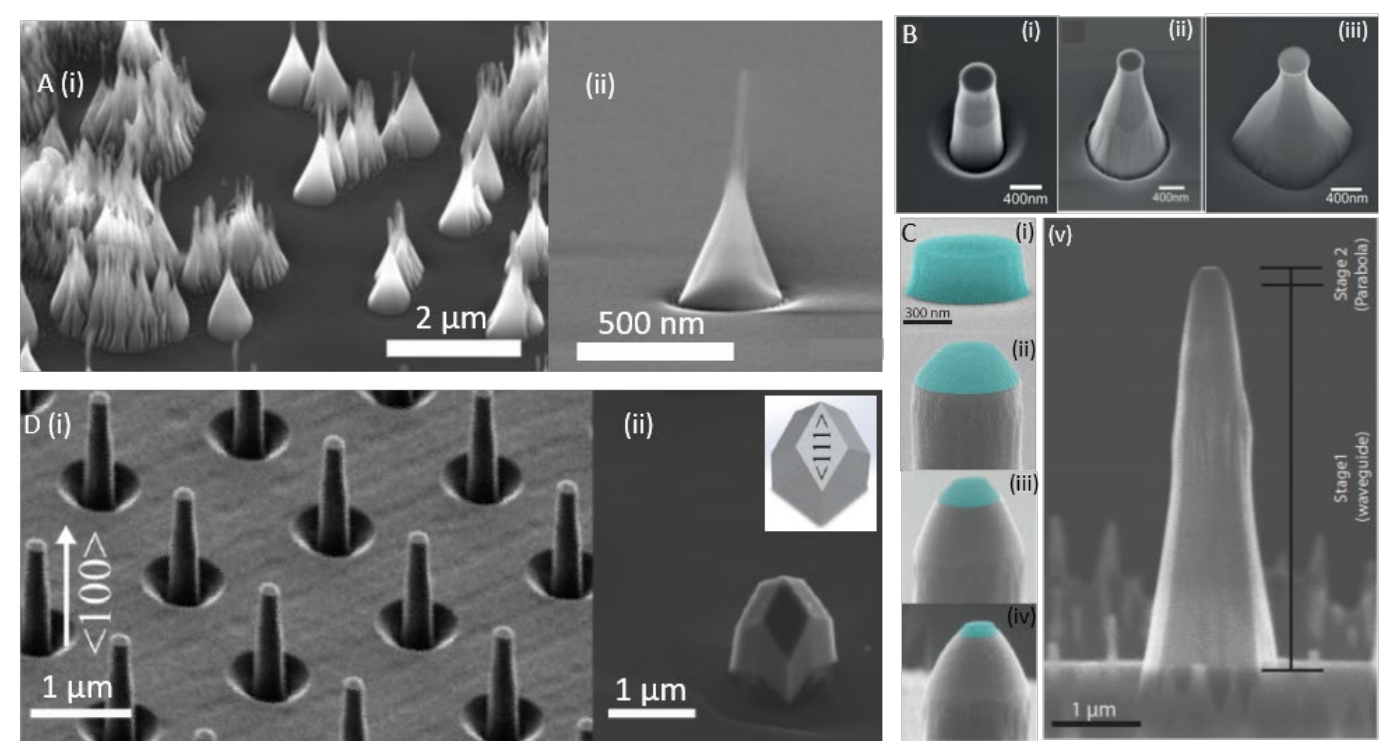

Figure 3. Scanning electron microscopy images of (A) (i) Tapered diamond nanopillars ((001)-oriented) with tip and conical base diameter of $15 \mathrm{~nm}$ and $200 \mathrm{~nm}$ patterned via ICP-RIE using silica bead masking layer over CVD grown diamond. (ii) High-resolution image of a single tapered nanopillar. Images reprinted with permission from Ref. [51] C 2020 WILEYVCH Verlag GmbH \& Co. KGaA, Weinheim; (B) Crystallographic orientation dependent etching of diamond to achieve nanopillars (top diameter of $350 \mathrm{~nm}$ and length of $1.5 \mu \mathrm{m}$ ) with tapering angles (half apex angle) of (i) $3.4^{\circ}$, (ii) $11.5^{\circ}$, (iii) $21^{\circ}$ obtained by varying the RF power during dry etching process. Images reprinted with permission from Ref. [52] (c) 2018 WILEY-VCH Verlag GmbH \& Co. KGaA, Weinheim; (C) Images depicting the fabrication process of diamond nanopillars with parabolic curvature, (i) $300 \mathrm{~nm}$ thick, $1 \mu \mathrm{m}$ wide disc shaped resist (FOX-16) mask patterned via E-beam lithography, (ii) first step of dry etching (ICP-RIE) to erode the mask at the edges, have trapezoidal cross-section with base diameter of $900 \mu \mathrm{m}$, (iii, iv) subsequent etching with controlled erosion of resist mask to obtain parabolic diamond surface, (v) Final nanopillar consisting of a parabolic tip with a $\approx 200 \mathrm{~nm}$ flat end-facet and long tapered waveguide region. Figure reprinted from Ref. [20] under creative commons CC BY 4.0 license; (D) (i) Cylindrical diamond nanopillars (200 nm diameter and $2 \mu \mathrm{m}$ length) fabricated using a combination of E-beam lithography and dry etching methods followed by (ii) an overgrowth step using microwave plasma enhanced CVD technique to obtain pyramidal pillars. Inset: schematic representation of the resulting pyramids with $<111>$ crystal facet. SEM images taken at $45^{\circ}$ angle. Figures reprinted from Ref. [53] within the open access publishing agreement of the Optical Society of America.

We summarize recent realizations of nanopillars and the respective fabrication methods in Table 1. The most common method involves pattern definition via EBL followed by highly directional etching using ICP-RIE. Alternative methods such as using silica beads as masks to minimize the dependence on lithography systems have been adapted [51]. In Figure 3A, tapered pillars obtained using this method are shown. However, the pillar density is challenging to control in this approach.

Nanopillars have been functionalized with a variety of color centers and various methods for color center creation are used: In addition to conventional ion implantation (before pillar fabrication) focused beams of ions allow for precise positioning (lateral and depth) into pre-structured nanopillars. For instance, Marseglia et al. [54], recently reported scalable fabrication of SCD nanowire arrays $(350 \mathrm{~nm}, 650 \mathrm{~nm}$ top and bottom diameter and height of $1.2 \mu \mathrm{m}$ ) via EBL and ICP-RIE followed by implantation of single or multiple $\mathrm{SiV}^{-} \mathrm{s}$ in individual nanowires using focused ion beams. In another approach, a homoepitaxial, $100 \mathrm{~nm}$ thick SCD layer with $\mathrm{SiV}^{-} \mathrm{s}$ on high purity type IIa diamond was patterned using EBL and RIE. The realized nanopillars host single SiV centers with high spectral stability [55]. In a similar approach, pre-structured SCD pillar were coated with spin-on-glass (perhydropolysilazane) and homoepitaxially overgrown via CVD method, to obtain localized ensembles of $\mathrm{SiV}^{-} \mathrm{s}$ in the overgrown part of nanopillars [56]. Creating color centers during growth potentially enhances their spectral stability as well as spin properties by avoiding implantation induced damage (see also Section 2.5) [55]. 
Table 1. Short description of different fabrication methods developed for nanowire/nanopillar realization.

\begin{tabular}{|c|c|c|c|}
\hline Methods Used & \multirow[t]{2}{*}{ Fabrication Method } & \multirow[t]{2}{*}{ Properties/Comments } & \multirow[t]{2}{*}{ References } \\
\hline Type/Dimensions & & & \\
\hline $\begin{array}{l}\text { SCD nanopillars with single tin } \\
\text { vacancy center: } \\
200 \mathrm{~nm} \text { diameter; } 500 \mathrm{~nm} \text { height }\end{array}$ & $\begin{array}{l}\text { - Preimplantation with } \mathrm{Sn} \\
\text { atoms } \mathrm{SnV}^{-} \text {depth of } 90 \mathrm{~nm} \\
\text { EBL \& ICP-RIE to fabricate } \\
\text { pillar pattern, Silicon nitride } \\
\left(\mathrm{Si}_{\mathrm{x}} \mathrm{N}_{\mathrm{y}}\right) \text { as hard mask }\end{array}$ & $\begin{array}{l}\text { - Obtained narrow emission } \\
\text { linewidths }(<250 \mathrm{MHz}) \text { of } \\
\text { single } \mathrm{SnV}^{-}\end{array}$ & [57] \\
\hline
\end{tabular}

SCD nanopillar:

Tapered with tip \& conical base diameter of $15 \mathrm{~nm} \& 200 \mathrm{~nm}$
- $\quad(100) \&(111)$-oriented CVD grown diamond

- Dilute Silica bead solution spread over diamond used as a mask for ICP-RIE
- $\quad$ Two step etching process: 1st to obtain nanopillars \& 2 nd to remove any organic/amorphous layer

- Density of nanopillars difficult to control
- Scalable fabrication method

- Nanowires with single or multiple $\mathrm{SiV}^{-} \mathrm{S}$

- Incorporation of single $\mathrm{SiV}^{-}$in nanowires yields higher light coupling efficiency (10 times) in comparison to single $\mathrm{SiV}^{-}$center in bulk diamond.
$350 \mathrm{~nm}$ top diameter, $650 \mathrm{~nm}$ bottom diameter \& height of $1.2 \mu \mathrm{m}$
- $\quad$ High purity, type IIa, HPHT SCD

- Nanowires created via EBL \& ICP-RIE

- $\quad$ Si ions implanted (AuSbSi source) via nanoimplanter at a penetration depth of $120 \mathrm{~nm}$
- $\quad$ (100)-oriented SCD

Overgrown SCD pillars with SiV ensembles: Tapered with diameter varying from 200-1000 nm \& height of $1 \mu \mathrm{m}$
- $\quad$ EBL and lift-off of deposited $\mathrm{Al}$ hard mask, ICP-RIE.

- Homoepitaxial overgrowth via CVD after spin coating with spin-on-glass
- $\quad$ Ensembles of $\mathrm{SiV}^{-} \mathrm{s}$ localised in the overgrown pillars

- $\quad$ Shape of overgrown part varies with initial diameter of the pillars: cubic for $500 \mathrm{~nm} \& 1000 \mathrm{~nm}$, pyramidal for $200 \mathrm{~nm}$ pillar
- $\quad$ High purity, type IIa SCD

SCD nanopillars with single $\mathrm{SiV}$ centers: $135-170 \mathrm{~nm}$ in diameter, $200 \mathrm{~nm}$ in height
- $100 \mathrm{~nm}$ thick homoepitaxial SCD layer containing in-situ created SiV centers

- $\quad$ Pattern HSQ layer via EBL \& RIE
- Optimized fabrication process to obtain single SiV per pillar

- High spectral stability of CVD grown $\mathrm{SiV}$ centers

\subsubsection{Diamond Inverted Nano-Cone (DINC)}

A novel waveguide type nano-structure allowing for efficient photon collection was recently reported by Jeon et al.: inverted nano-cones (DINCs) were realized in high quality SCD [58]. NV centers (depth of NVs approx. $200 \mathrm{~nm}$ ) were created via ion implantation prior to fabricating DINCs using EBL and two step RIE. First, anisotropic, vertical $\mathrm{O}_{2}$ plasma etching was carried out using a silicon nitride hard mask, to control the height of the nano-cone. Subsequently, angled dry etching leads to the conical shape of DINCs. To allow circularly symmetric, smooth etching of DINCs, a cone shaped Faraday cage is used. Figure 4A(i) shows the DINC's with top and bottom diameters of $550 \mathrm{~nm}$ and $50 \mathrm{~nm}$, respectively. The inverted taper angle of the DINCs is 78.5 degrees. Because of ion channeling, the created NV centers have a wide depth distribution within the DINC's. Consequently, statistical analysis of photon collection efficiency of DINCs, conventional diamond nanopillars and bulk substrates is necessary to evaluate the enhancement. The DINCs showed enhanced photon collection efficiencies compared to conventional diamond nanopillars. Moreover, DINCs are easily detachable from the substrate owing to the small 
cross-sectional area at the bottom and relocated to other substrates with high precision. Figure $4 \mathrm{~A}(\mathrm{ii})$ illustrates the transfer of a single DINC onto Au chip using a tungsten tip.
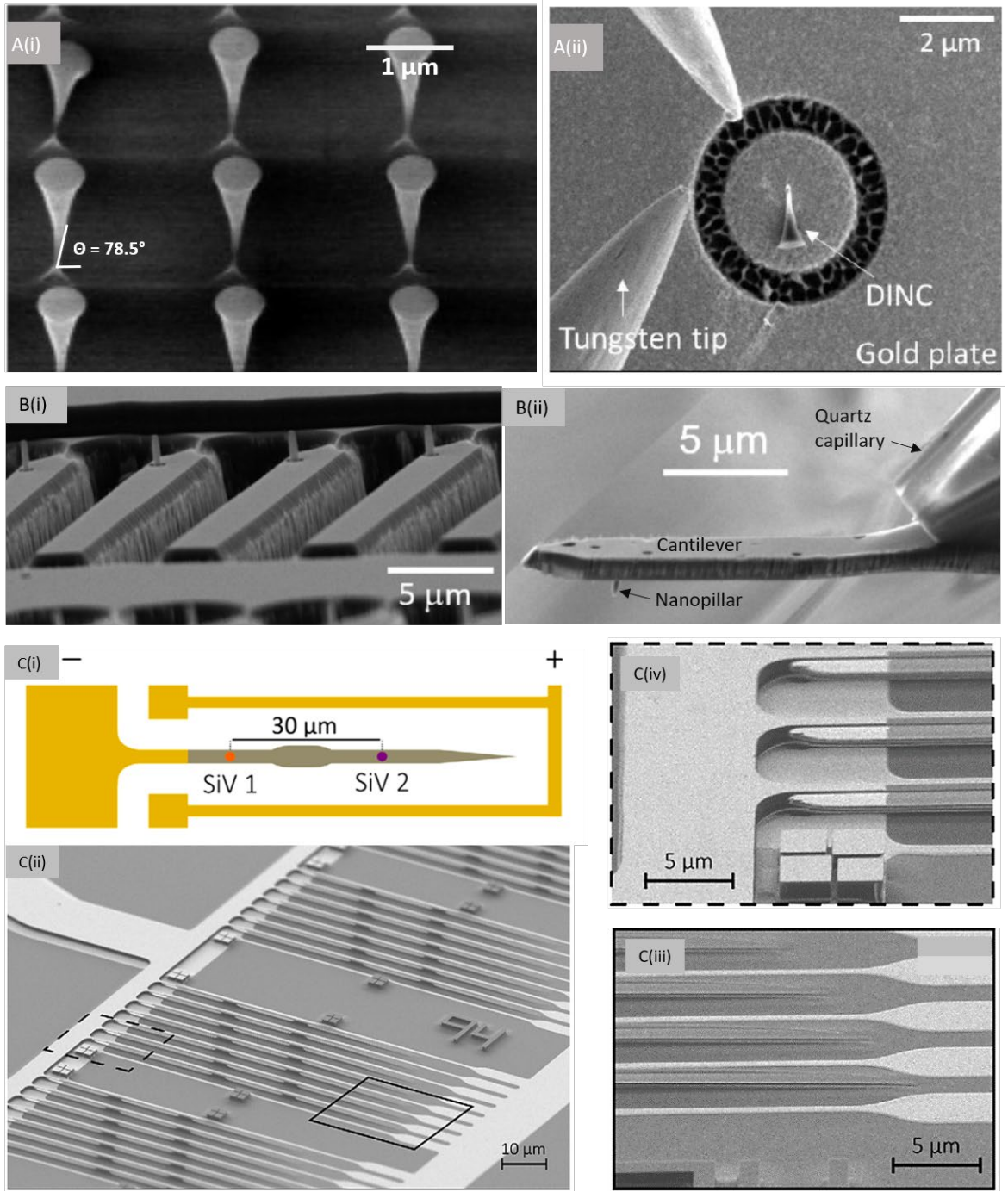

Figure 4. SEM images of (A) (i) Diamond inverted nano-cones (DINC) realized via a combination of Ebeam lithography and angled dry etching (ICP-RIE) using a conical faraday cage. (ii) Transfer of DINC onto a gold target plate using tungsten tips for coupling of DINCs to other photonic components. Reprinted (adapted) with permission from Ref. [58]. Copyright (2020) American Chemical Society. (B) (i) An array of monolithic diamond scanning probes fabricated using multiple E-beam lithography and dry etching processes. The shown nanoprobes required back side diamond etching for release of the devices. (ii) Final released device consisting of a cantilever with nanopillar, attached to a quartz capillary which acts as a holder for mounting the device to a scanning probe microscope. Images reprinted from Ref. [59] under creative commons CC BY 4.0 license. (C) (i) Schematic representation of a diamond nanophotonic device where the gray region represents the diamond waveguide implanted with $\mathrm{SiV}$ centers at defined locations, while the yellow stripes represent the electrodes to form a capacitor. On application of bias voltage between the device (-ve terminal) and the bottom (+ve terminal), the doubly clamped cantilever is deflected and results in localized tuning of the color center. (ii) SEM image of the photonic device realized using a combination of E-beam lithography and angled etching technique of diamond substrate. Subsequent lithography steps were carried out to implant precisely implant $\mathrm{Si}^{-}$ions (to form $\mathrm{SiV}$ centers) and lift-off process for electrode definition. (iii) Capacitor plates defined on and below the devices. (iv) Tapered diamond to efficiently extract photons from waveguides. Images reprinted from Ref. [39] under creative commons license CC BY 4.0. 


\subsection{Scanning Probes Based on Color Centers in Diamond Nano-Structures}

$\mathrm{NV}$ center based sensors offer impressive sensing capabilities for magnetic and electric fields $[2,60]$. To allow for nanoscale spatial resolution, it is important to bring the NV center and the sample of interest as close as $100 \mathrm{~nm}$ or less. This requires using a scanning probe structure. Table 2 summarizes the approaches to create such structures with scannable $\mathrm{NV}$ centers including attachment of diamond nanocrystals to conventional AFM tips and structuring of monolithic SCD scanning probes. In an alternative approach, the sample is attached to the cantilever of a commercial scanning probe microscope and scanned over an $\mathrm{NV}$ embedded $\approx 5 \mathrm{~nm}$ below an SCD surface and is equivalent to scanning of diamond probes over the sample surface [61,62]. Ernst et al. [63] recently demonstrated another method of using planar SCD (50 $\mu$ m thick) with single NV centers as scanning probes for imaging planar samples, which required highly precise alignment (radial) and proximity control between sensor and sample. While initial scanning probes relied on grafting color center containing diamond nanocrystals to AFM tips, the focus recently shifted to realization of top-down fabricated SCD nanoprobes [20,60]. They have several advantages: robust and usable in harsh environments (e.g., cryogenic temperatures), not limited by optical bleaching and excess spin dephasing and the ability to tailor the shape of the diamond tip to enhance photon collection [20].

All SCD scanning probes are fabricated using multiple steps of EBL and RIE processes. An example of an array of fabricated nanoprobes is illustrated in Figure $4 \mathrm{~B}(\mathrm{i})$. The probes are mounted to a holder e.g., a capillary for integration into an AFM system as shown in $4 \mathrm{~B}(\mathrm{ii})$. Most scanning probes developed till date comprise of truncated cones with blunt, circular end-facet of $\sim 200 \mathrm{~nm}$ diameter [53]. Though such tips have beneficial photonic properties, their blunt facet prevents obtaining high resolution AFM imaging in parallel to e.g., magnetometry. Moreover, the blunt tips require precise angular alignment to not create an additional, unwanted distance between color center and sample.

Tailoring the scanning probe geometry can enhance photon collection, AFM capability and spatial resolution. Nanopillars with large taper angles obtained via plane dependent dry etching enhance photon collection due to the combined effect of optical wave guiding and adiabatic changes of the effective refractive index [52]. Figure 3A,B show pillars with various taper angles. Such tapered pillars also improve the mechanical strength of the probe. In another approach, nanopillars with parabolic curvature $(200 \mathrm{~nm}$ flat-end facet) and long tapering waveguide section achieve 5-fold increment in photon collection efficiency from a near surface NV [20]. The parabolic shape is achieved via a two-step ICPRIE process shown in Figure 3C, in which the gas mixture is changed to obtain a parabolic end facet with long tapering waveguide section. Giese et al. [64] recently reported high aspect-ratio $(>60)$, triangular diamond nanobeams fabricated via Faraday cage angled etching, integrated into an AFM setup. This approach has the advantage that it omits the preparation of thin membranes. Integrating NVs at the extremity of such nanobeams allows high photon collection efficiency along with high scanning resolution of structures with steep sidewalls.

One route to optimized nanopillar scanning probes might be to combine top-down and bottom-up methods to develop pyramidal probes [53]. To this end, Batzer et al. used cylindrical nanopillars (diameter $=200 \mathrm{~nm}$ and length $=2 \mu \mathrm{m}$ ) fabricated using EBL and ICP-RIE on (100)-oriented SCD [65]. Figure 3D(i), shows an image of those cylindrical pillars. The pillars were then overgrown using microwave plasma enhanced CVD, creating smooth and sharp pyramidal tips with tip radii of $10 \mathrm{~nm}$ (see Figure 3D(ii)). Such sharp pyramidal tips with near surface color centers are promising for high-resolution AFM imaging, combined with enhanced spatial resolution color center based sensing, photonic properties and long spin coherence time. Moreover, also for scanning probes, recently the more advantageous (111) diamond orientation has been used [66]. 


\subsection{General Aspects of Color Center Creation, Surface Termination and Tuning}

Before proceeding to nanomechanical structures, we here summarize some aspects of color center creation and surface termination, relevant for the discussed nano-structures. For various quantum applications, shallow NV centers less than roughly $50 \mathrm{~nm}$ below the surface are necessary. Single NVs are preferred for high spatial resolution sensing, e.g., scanning probe techniques, however, the fluorescence signal is weak. In contrast, ensembles of NVs can give better sensitivity and fast imaging of a large area of interest with reduced spatial resolution [50,67]. Shallow NV centers are created using low energy ion implantation and delta-doping often combined with electron irradiation, or implantation of helium ions $[68,69]$. Although these methods have high positioning accuracy, they are limited in conversion efficiency (only a few \% or less) of implanted nitrogen creates NV centers (yield) [70]. Part of the created vacancies are lost in recombination with carbon interstitials, vacancy complex formation (di-vacancies, vacancy chains) or diffusion to the diamond surface [71]. Additionally, shallow, implanted NVs have relatively short spin coherence time ( $\mathrm{T}_{2} \sim 10 \mu \mathrm{s}$ in comparison to $600 \mu \mathrm{s}$ for NVs in bulk), and exhibit instability of the negatively charged state of $\mathrm{NV}^{-}[72,73]$. It is mainly attributed to the implantation induced damage formed in the diamond lattice in close vicinity to the implanted NV centers, which is not fully eliminated post thermal annealing.

Thus, in addition to the fabrication of nano-structures, optimized methods to create shallow color centers are central for quantum technologies. These include optimization of the thermal annealing condition after nitrogen implantation, treatment in soft oxygen plasma and additional annealing steps in oxygen environment. Annealing in oxygen environment at $450{ }^{\circ} \mathrm{C}$ enhances the surface ordering at the atomic scale thereby enhancing $\mathrm{NV}$ coherence, which is limited below tri-acid cleaned surfaces as the oxygen groups are highly disordered [72]. Moreover, oxygen annealing stabilizes the negatively charged state of shallow NVs [73]. Significant improvement in $\mathrm{T}_{2}$ (10-fold) was achieved using a thin, $p$-doped diamond layer (e.g., boron) epitaxially grown on ultrapure diamond, prior to nitrogen implantation. This approach charges vacancies and prevents vacancy cluster formation during annealing, consequently enhancing the NV formation yield [70]. In another approach, Herbschleb et al. reported usage of high-quality $n$-doped diamond with phosphorus atoms to improve NV based sensing [74]. The highest NV center yield (75\%) with enhanced $\mathrm{T}_{2}$ was reported in sulphur-doped diamond ((100)-CVD grown) which also provides highest $\mathrm{NV}^{-}$stability.

The influence of diamond surface termination via multiple atoms such as oxygen, hydrogen, fluorine, silicon and nitrogen on the charge stability of shallow implanted $\mathrm{NV}^{-} \mathrm{s}$ has been studied [72,73]. It has been found that the surface termination with negative electron affinities (NEA) such as on termination with hydrogen or silicon, results in instability of the charge state of shallow NVs. On the other hand, termination of surfaces with positive electron affinities (PEA) for instance with oxygen or fluorine, enhances the stability of $\mathrm{NV}^{-}$s. In a recent report, Kawai et al. investigated the influence of $\mathrm{Si}$ and $\mathrm{N}_{2}$ termination on the stability of shallow implanted $\mathrm{NV}^{-}$s. The termination with $\mathrm{Si}$ and $\mathrm{N}_{2}$ was carried out by silicon evaporation at high temperature $\left(950^{\circ} \mathrm{C}\right)$ and nitrogen radical beam exposure, respectively on high purity diamond grown over (001)-oriented diamond substrate. It was found that $\mathrm{Si}$ termination of the surface yielded unstable $\mathrm{NV}^{-} \mathrm{s}$, while nitrogen terminated surfaces (full $n$ or $\mathrm{N} / \mathrm{H}$ ) stabilized $\mathrm{NV}^{-} \mathrm{s}$ with positive surface electron affinity and spin properties (e.g., coherence time) comparable to that of oxygen terminated surfaces.

In addition to charge state instabilities and reduced coherence, especially color centers in nano-structures can show a spread in transition frequencies which makes their use especially in quantum information challenging. Recently, nano-structures have been presented that directly implement local tuning of color center transitions via strain. Figure $4 \mathrm{C}(\mathrm{i})$ shows such structures, in which the grey region represents a waveguide section with $\mathrm{SiV}$ centers implanted at defined locations, while the yellow stripes represent metallic contacts forming a capacitor [39]. The doubly clamped cantilever is deflected on application of a 
bias voltage between the device (-ve terminal) and the bottom (+ve terminal) and leads to localized strain tuning of the $\mathrm{SiV}$ centers. Figure $4 \mathrm{C}$ (ii) shows an SEM image of such a device. These devices were realized using a combination of EBL and angled etching. Further lithography processes were implemented to precisely implant $\mathrm{Si}^{-}$ions (to form $\mathrm{SiV}$ centers) and to define metallic electrodes via a lift-off process. The inset Ciii shows the waveguide taper for efficient extraction of photons, while Civ represents the capacitor plates patterned on and below the devices.

Table 2. Examples of different shapes/assemblies of diamond for scanning probe magnetometry applications.

\begin{tabular}{|c|c|c|c|c|}
\hline Properties & Shape \& Dimensions of & Fabrication Method & Comments & References \\
\hline Type of Scanning Probe & & & & \\
\hline \multirow{5}{*}{$\begin{array}{c}\text { All diamond } \\
\text { scanning probe } \\
\text { (100)-oriented diamond. } \\
\text { NV orientation and } \\
\text { magnetic field sensing is } \\
\text { along an axis tilted by } \\
54.7^{\circ} \text { from } \\
\text { nanopillar direction. }\end{array}$} & $\begin{array}{c}\text { Nanopillar: } \\
\text { diameter }=350 \mathrm{~nm} ; \\
\mathrm{L}=3.5 \mu \mathrm{m} \\
\text { Cantilever probes: } \\
\mathrm{L}=125 \mu \mathrm{m}, \mathrm{W}=\mathrm{H}=50 \mu \mathrm{m}\end{array}$ & $\begin{array}{l}\text { EBL \& ICP-RIE for nanopillar } \\
\text { structuring with } 10 \mathrm{~nm} \text { Ti as } \\
\text { adhesion layer on diamond; } \\
\text { UVL for cantilever probe } \\
\text { definition; deep etch with } \\
400 \text { nm Ti as an etch mask. NV } \\
\text { depth defined using } \\
\text { ion implantation. }\end{array}$ & $\begin{array}{l}\text { Robust, simple fab. process } \\
\text { for } 52 \text { probes on } \\
2 \mathrm{~mm} \times 4 \mathrm{~mm} \text { substrates } \\
\text { Integrated RF components } \\
\text { on the cantilever probes }\end{array}$ & [19] \\
\hline & $\begin{array}{l}\text { Triangular shaped beam: } \\
\mathrm{L}=30 \mu \mathrm{m}, \mathrm{W}=450 \mathrm{~nm}\end{array}$ & $\begin{array}{l}\text { EBL for patterning with } \mathrm{Ni} \text { as } \\
\text { protective mask for structuring } \\
\text { diamond; Faraday cage used for } \\
\text { ICP-RIE to obtain high } \\
\text { aspect-ratio triangular } \\
\text { shaped beam. }\end{array}$ & $\begin{array}{l}\text { High aspect-ratio of tip } \\
\text { suitable for scanning of } \\
\text { structures with } \\
\text { steep sidewalls } \\
2000 \text { devices possible on } \\
4 \mathrm{~mm} \times 4 \mathrm{~mm} \text { substrates } \\
\text { - } \quad \text { Location of NVs not defined }\end{array}$ & {$[64]$} \\
\hline & $\begin{array}{l}\text { Pillar with parabolic } \\
\text { curvature: } 200 \text { nm flat-end } \\
\text { facet, long tapering } \\
\text { waveguide section }\end{array}$ & $\begin{array}{l}\text { Cantilever \& pillar definition } \\
\text { using EBL followed by ICP-RIE } \\
\text { etching. A deep etch is carried } \\
\text { out for releasing the diamond in } \\
\text { the end. }\end{array}$ & $\begin{array}{l}\text { Parabolic design yields high } \\
\text { photon collection efficiency } \\
\text { from a near surface NV. }\end{array}$ & {$[20]$} \\
\hline & $\begin{array}{l}\text { Diamond bonded on } \\
\text { oxidized Si. Pillar: } 200 \mathrm{~nm} \\
\text { diameter, } 1 \mu \mathrm{m} \text { length; } \\
\text { cantilever: } 150 \times 20 \times 3 \mu \mathrm{m} \text {; } \\
\text { Array of pillars }(1 \mu \mathrm{m} \text { pitch }) \\
\quad \text { on each cantilever }\end{array}$ & $\begin{array}{l}\text { Pillar structuring using NIL with } \\
\text { Ti hard mask and } \mathrm{O}_{2} \text { etching. } \\
\text { The cantilevers released using } \\
\text { deep RIE through Si substrate. } \\
\text { Single NV per pillar at } \\
\text { 15-20 nm depth. }\end{array}$ & $\begin{array}{l}100 \text { cantilevers on } \\
2 \text { mm } \times 2 \text { mm diamond. } \\
\text { The unconventional AFM } \\
\text { cantilever with multiple tips } \\
\text { (pillars) favours the } \\
\text { probability of finding } \\
\text { shallow NV pillar } \\
\text { with favourable } \\
\text { coherence properties } \\
\text { Wide-field imaging possible } \\
\text { for large areas to obtain } \\
\text { vector field magnetometry }\end{array}$ & {$[18]$} \\
\hline & Nanopillar: 200 nm diameter & $\begin{array}{l}5 \mu \mathrm{m} \text { thick diamond membrane } \\
\text { creation using shadow mask \& } \\
\text { RIE; EBL \& RIE for definition of } \\
\text { nanopillar \& cantilever with } \\
5 \mathrm{~nm} \text { Ti as adhesion layer used } \\
\text { for HSQ on diamond; and } \\
\text { pre-patterned alignment marks } \\
\text { using EBL }\end{array}$ & $\begin{array}{l}\text { - } \quad \text { No ICP used, only RIE } \\
\text { Fabrication of around } 1000 \\
\text { cantilevers possible on } \\
2 \mathrm{~mm} \times 2 \mathrm{~mm} \text { sample }\end{array}$ & {$[75]$} \\
\hline $\begin{array}{l}\text { Bulk diamond with NV } \\
\text { centre for imaging sample } \\
\text { scanned on top }\end{array}$ & $\begin{array}{l}\text { Single NV centers } \\
\text { embedded } 5 \mathrm{~nm} \text { below type } \\
\text { Ila diamond membrane of } \\
30 \mu \mathrm{m} \text { thickness }\end{array}$ & $\begin{array}{l}\text { Shallow implantation of single } \\
\text { NV centers at } 2.5 \mathrm{keV} \text { using } \\
{ }^{15} \mathrm{~N}^{+} \text {ions }\end{array}$ & $\begin{array}{l}\text { - Only possible if sample can } \\
\text { be attached to cantilever }\end{array}$ & {$[61]$} \\
\hline $\begin{array}{l}\text { Diamond nanocrystals } \\
\text { with single NV center } \\
\text { attached to conventional } \\
\text { AFM tips }\end{array}$ & $\begin{array}{c}\text { Nanodiamonds }(20 \mathrm{~nm}) \\
\text { with single NV grafted on } \\
\text { AFM tips }\end{array}$ & $\begin{array}{l}\text { Diamond nanocrystals } \\
\text { synthesized by milling of type } \\
\text { 1b HPHT diamond crystals with } \\
\text { high } \mathrm{N}_{2} \text { content. }\end{array}$ & $\begin{array}{l}\text { - Limited in sensing } \\
\text { performance due their short } \\
\text { spin coherence times }\end{array}$ & {$[76]$} \\
\hline
\end{tabular}




\section{Nanomechanical Components}

Nanomechanical components such as cantilevers have a very small mass and are highly responsive to changes in their local environment. Applications of nanomechanical components include scanning probe magnetic and atomic force microscopy that yields attonewton level sensitivities as well as the detection of the mass of single molecules and proteins in real-time [1,77].

The minimum detectable force $F_{\min }$ using a nanomechanical cantilever is limited by thermomechanical noise which is governed by dissipation of mechanical energy and absolute temperature (T) [1]:

$$
F_{\text {min }}=\sqrt{\frac{2 k_{B} T B k_{c}}{\pi f_{c} Q_{m}}}
$$

where $k_{c}, f_{c}, k_{B}$ represent the spring constant, resonant frequency, and Boltzmann constant respectively. $Q_{m}$ represents the mechanical quality factor and characterizes the bandwidth of the resonator (i.e., $B$ ) with respect to $f_{c}$. High $Q_{m}$ values indicate low rates of loss of mechanical energy. For a rectangular cantilever with no-internal stress, $F_{\min }$ can be described using thickness $(t)$, width $(w)$, length $(l)$, mass density $(\rho)$ and Youngs modulus $(E)$ of cantilever [1]:

$$
F_{\text {min }}=\sqrt{\frac{w t^{2} k_{B} T B}{l Q_{m}} \sqrt{E \rho}}
$$

To enhance the sensitivity of the cantilever, it is required to decrease the mass and keep the cantilever narrow, thin and long. Several efforts have been made to develop thinner cantilevers with lower $k_{c}$ 's in conventional materials such as $\mathrm{Si}$ or by fabricating doubly clamped beams. However, reducing the thickness of nanocantilevers leads to decrease in $Q_{m}$ due to increase in the surface friction by surface defects. This issue is overcome by using cryogenic temperatures which limits the practical applications of such resonators.

The huge interest in realizing nanomechanical components based on diamond is due to its outstanding mechanical and thermal properties and the possibility to embed spin-carrying color centers into nanomechanical components. Diamond's record high bulk Young modulus provides high mechanical resonance frequencies and low mechanical losses. Also, its high thermal conductivity and low thermal expansion coefficient allows high dissipation of heat. Color centers enable optical readout of nanoresonator motion with the capability of magnetic sensing and to implement optomechanics on the same platform. These properties are retained at room temperature and with the known biocompatibility of diamond, nanoresonators are suitable for sensitive detection of living cells/tissues.

Diamond nanoresonators are fabricated using the top-down methods described in Section 2 for photonic components. Commercially available diamond membranes are transferred to carrier substrates for easy handling and patterned using a combination of lithography and RIE. In the following subsections, we briefly describe advanced diamond nanoresonators realized in recent years and discuss their advantages.

\subsection{Nanocantilevers}

Diamond nanocantilevers and nanobeams are used for ultrahigh force sensitivity. Nanocantilevers are clamped on one end while the beams are clamped on both the ends. Commercial diamond substrates are pre-processed using RIE and polishing to achieve low surface roughness $(<5 \mathrm{~nm})$ and high thickness uniformity (better than $1 \mu \mathrm{m}$ variation over $\mathrm{mm}$ sized sample) to reliably realize cantilever/nanobeam resonators via lithography and RIE.

Top-down fabricated nano-resonators have low device-device variability. According to Equation (3) $\left(F_{\min }\right.$ the sensitivity of nanomechanical sensors is limited by thermomechanical force noise, which is reduced for structures with low spring constant $k_{c}$ and mass, m. Tao et al. developed rectangular cantilevers with high $Q_{m}\left(>10^{6}\right.$ at $\mathrm{mK}$ temperatures) by fabrication using robust diamond on insulator or quartz bonded substrates [77]. Additionally, functionalization of cantilever surfaces with oxygen and fluorine groups 
has shown to positively affect the surface friction (decrease in surface friction due to cleaner and better defined surface groups) and thus influence the $Q_{m}[77,78]$. In a recent report by Eichler et al. [12], nanoladder configuration cantilevers are used to decrease the cross-section while maintaining the cantilever length thereby keeping the $k_{c}$ and $\mathrm{m}$ low. The nanoladder cantilever consists of two parallel nanowires $(200 \mathrm{~nm}$ wide and $150 \mu \mathrm{m}$ long) connected by struts ( $200 \mathrm{~nm}$ wide, $3 \mu \mathrm{m}$ long) along their length and was realized using a combination of EBL and ICP-RIE. Figure 5A shows the nanoladder cantilever with zoomed in-view of individual regions (base, middle and tip regions) in the insets. Highest $Q_{m}$-factors measured with the realized nanoladder cantilever at $\mathrm{mK}$ temperatures were 162,000 .

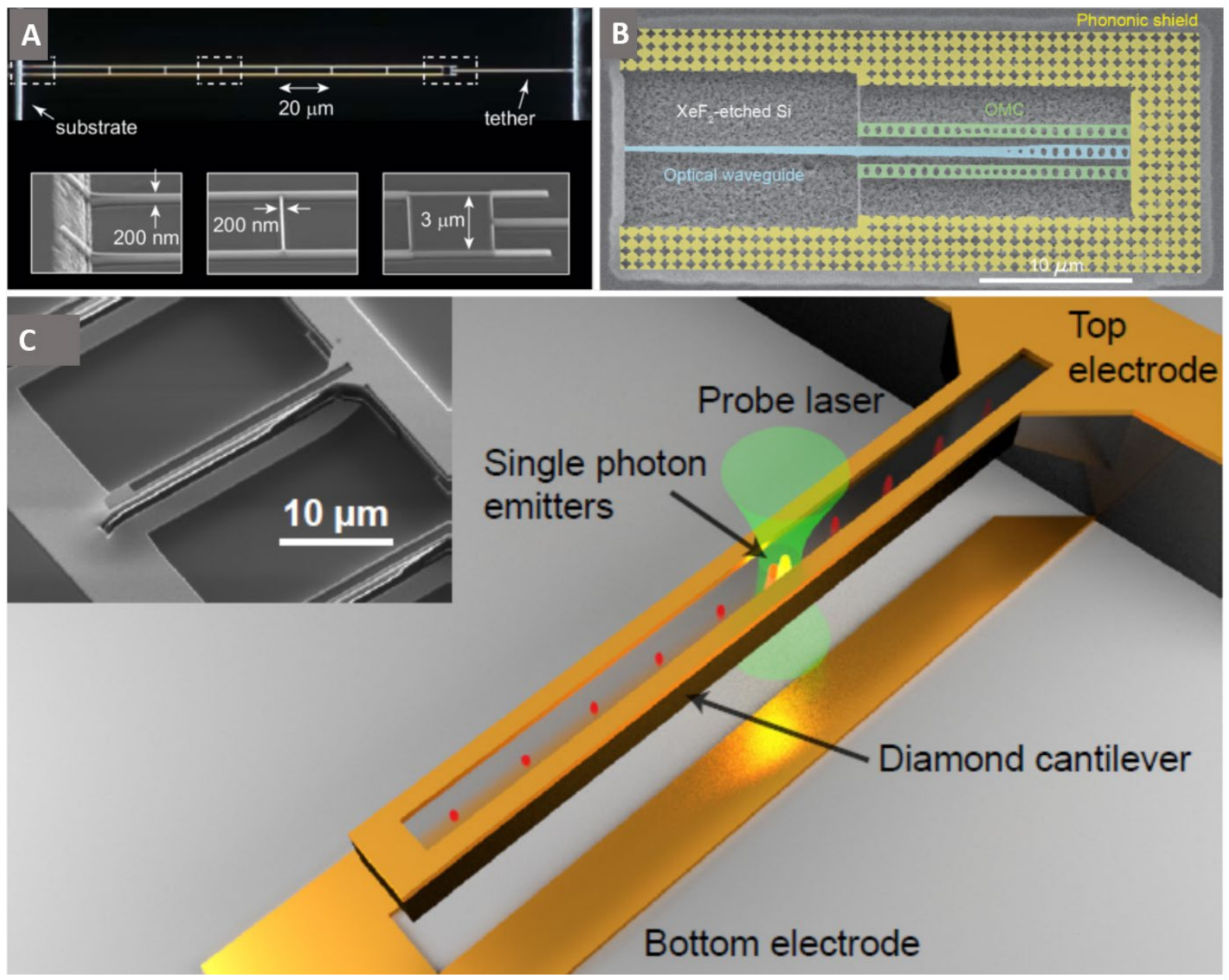

Figure 5. (A) Optical micrograph of a single crystal diamond nanoladder cantilever (150 $\mu \mathrm{m}$ long) realized using a combination of E-beam lithography and dry etching processes. Each nanoladder device consists of two parallel nanowires (200 $\mathrm{nm}$ wide) connected via rugs at every $20 \mu \mathrm{m}$ distance. The zoomed in view of the base, middle and tip regions of the ladder is shown in the insets. Reprinted (adapted) with permission from Ref. [12]. Copyright (2018) American Chemical Society. (B) SEM image of a pair diamond optomechanical crystals on either side of diamond optical waveguide realized using diamond on insulator technique. The optomechanical crystal consists of a rectangular cross-section, single crystal diamond nanobeam with a one-dimensional array of ellipses etched along its length. Image reprinted with permission from Ref. [28] copyright IOP publishing, Reproduced with permission. All rights reserved. (C) Schematic representation of a diamond microcantilever realized using a combination of E-beam lithography and angled etching technique followed by electrode definition using e-beam lithography and lift-off process. The color centers $(\mathrm{GeV})$ are precisely implanted (lateral accuracy $>50 \mathrm{~nm}$ ) using focussed ion beam implantation technique. Application of a bias voltage between the top and bottom electrodes causes strain in the cantilever. Using a probe laser, multiple color centers (GeVs) can be simultaneously optically accessed. SEM image of the diamond microcantilever with dark and light regions representing the diamond and electrodes is shown in the inset. Reprinted with permission from Ref. [79]. Copyright (2018) by the American Physical Society. 


\subsection{Nanowires}

Diamond nanowires integrated with $\mathrm{Si} /$ diamond cantilever are used for sensitive force detection in scanning force microscopy. Owing to its low dielectric constant, diamond has low non-contact friction which normally deteriorates force sensitivities when the tip is too close to the surface [1]. The realization of nanowires (with different diameters and lengths) for force sensing applications is carried out by methods described earlier for fabrication of nanopillars.

\section{Optical and Mechanical Hybrid Systems}

Hybrid mechanical systems in which quantum elements such as qubits are coupled to mechanical oscillators attracted significant attention in recent years due to their potential in addressing key issues in quantum information processing, communication and control. Cady et al. recently realized a diamond optomechanical crystal (OMC) with embedded NV centers via the diamond on insulator technique [28]. The OMC design consists of an SCD nanobeam with a rectangular cross-section in which a one-dimensional array of ellipses is etched via ICP-RIE. Figure 5B shows a pair of SCD OMCs on either side of an SCD optical waveguide, surrounded by a phononic shield. The NV center interacts with the mechanical motion of the OMC through strain in the crystal. The light emitted by the NV center is measured using a confocal microscope and the OMC is probed through coupling to the adjacent optical waveguide. The rectangular cross-section OMCs are promising for hybrid NV-mechanical systems owing to the z-symmetric strain profile of the fundamental breathing acoustic mode with the feasibility to realize 2-dimensional phononic shields, paving the way for high mechanical quality factors. Maximum Quality factors of 42000 were achieved with the realized OMCs, which further need to be improved (to reach $Q \sim 10^{6}$ ) in addition to NV center spin coherence to enhance coupling between an NV center spin and mechanical motion of OMC.

Color centers in diamond are important components of optical quantum technologies, however, photons from different emitters are often spectrally distinguishable due to the complex mesoscopic environments of the color centers. Strain-based spectral alignment of two solid-state single photon emitters was achieved by Maity et al., using the strain gradient in diamond microcantilevers [79]. The diamond microcantilever in Figure 5C was fabricated using a combination of EBL and angled-etching. Subsequently, EBL and lift-off were implemented to define the electrodes. $\mathrm{GeV}$ color centers are implanted precisely via focused ion beam implantation. An SEM image of the microcantilever is shown in the inset with dark and light regions representing the diamond and the electrodes, respectively. The cantilever is strained by a bias voltage between the top and bottom electrodes and multiple GeV's can be simultaneously accessed optically using a probe laser. The developed mechanism paves the way to realize identical quantum emitters for on-chip integrated quantum systems.

Moreover, conversion between different colors of light is important for coupling quantum optical technologies which have different optical wavelengths. Optomechanical conversion of wavelengths and amplification has been recently demonstrate in SCD microdisk cavities by Mitchell et al. [80]. The realized devices are promising for converting the emission of diamond color centers to telecommunication wavelengths for applications in quantum networks.

\section{Other Diamond Nano-Structures}

\subsection{Diamond Mirrors}

Optical components such as mirrors which withstand extremely high laser powers are desired to steer the laser to the target location in applications varying from laser manufacturing, semiconductor industry and medicine [46]. Conventional mirrors consist of multilayer coatings of thin dielectric layers of different refractive index and thickness. Defects and imperfections in the multilayer dielectric coatings lead to absorption of laser energy at high powers, thereby causing thermal stress and permanent damage. These 
limitations were overcome by realizing monolithic diamond mirrors which are not limited by thermally induced damage. Diamond mirrors created by etching nano-structures into diamond surfaces, exhibited favorable optical properties with extremely high thermal conductivity. An optical image of diamond mirrors (on $4.2 \mathrm{~mm} \times 4.2 \mathrm{~mm}$ diamond chip) fabricated using a combination of EBL and RIBAE is illustrated in Figure 6A(i). Each division on the ruler measures $1 \mathrm{~mm}$. In Figure 6A(ii), SEM image of the diamond mirror taken at $60^{\circ}$ angle is shown with zoomed in view (iii) depicting golf tee features of the mirror taken at $40^{\circ}$ angle. The realized diamond mirrors show high reflectivity $(>98 \%)$ and are capable of withstanding extremely high laser powers.
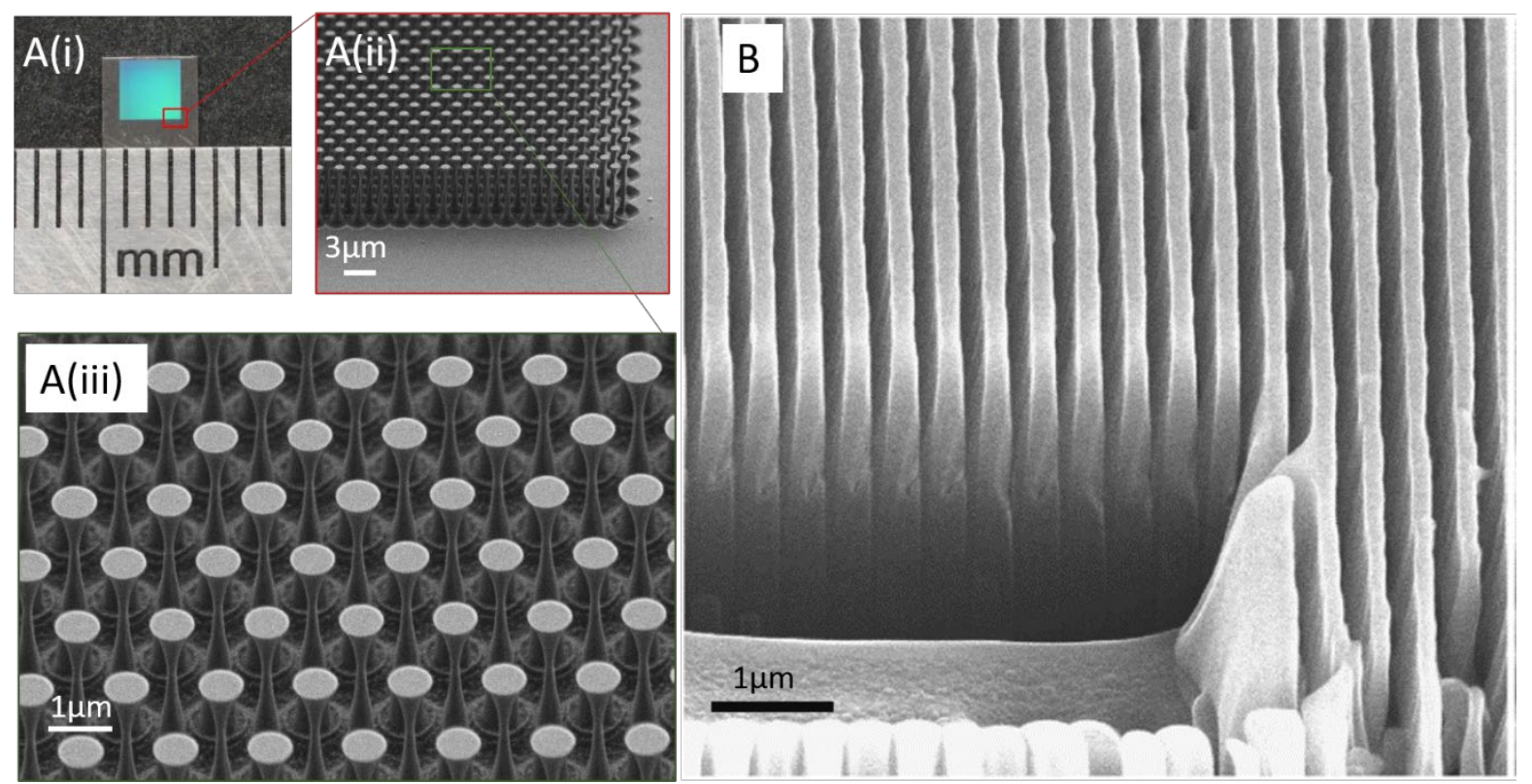

Figure 6. (A) (i) Optical microscope image of diamond mirrors (on $4.2 \mathrm{~mm} \times 4.2 \mathrm{~mm}$ diamond chip) fabricated via reactive ion beam angled etching. Each division on the ruler measures $1 \mathrm{~mm}$. A (ii) SEM image of the diamond mirror taken at $60^{\circ}$ angle with zoomed in view A (iii) showing golf tee features of the mirror taken at $40^{\circ}$ angle. Reprinted with permission from the author from Ref. [46]. (B) SEM image of $400 \mathrm{~nm}$ pitch diamond nanogratings fabricated via a combination of interferometric lithography and dry etching. Figure reprinted from Ref. [81] under creative commons CC BY 4.0 license.

\subsection{Diamond Nanogratings}

Sensors based on NV centers are promising for low volume NMR spectroscopy, but are limited in sensitivity [81]. Kehayias et al. recently reported a method to enhance the surface area of such sensors via nano-structuring of dense high-aspect-ratio nanogratings. In Figure 6B, an SEM image of such diamond nanogratings with $400 \mathrm{~nm}$ pitch realized using a combination of interferometric lithography and RIE is shown. The sidewalls of the nanogratings are doped with NV centers few nanometers below the diamond surface and are deployed for NMR spectroscopy in $1 \mathrm{pL}$ volume of solution placed adjacent to nanograting grooves at room temperature. Using the realized diamond nanogratings, approximately two orders of magnitude improvement in concentration sensitivity from previously reported NV and picolitre NMR studies was obtained.

\section{Conclusions and Outlook}

In this review, we summarized advanced nano-structuring methods for diamond. The different top-down fabrication methods mainly comprising of lithography and dry-etching processes are described. In recent years, various novel configurations of nano-structures with applications varying from photonics, mechanics, optomechanics, nanoelectronics, mirrors etc. were realized and are reviewed here. The methods for precise and controlled 
creation of color centers such as NVs or group-IV defects for specific applications are also discussed.

Though the advancement in nanofabrication methods resulted in novel nano-structures with superior photonic or/and mechanical properties there are certain limitations which are hindering up-scaling the processes. The growth of wafer-scale, high quality, single crystal diamond is limited, while nano-structuring of individual diamond substrates often results in device-to-device variability. Optimal processes are still being developed: e.g., the commonly used e-beam resist HSQ adheres poorly to diamond and thus suitable adhesion promoting layers such as $\mathrm{Si}$ or $\mathrm{SiO}_{2}$ are required [41,59]. Additionally, HSQ is highly sensitive to oxygen in air with low shelf life, leading to very limited stability after coating. This limits batch processing of diamond nano-structures. Moreover, selectivity of HSQ based masks to oxygen plasma etching is imperfect, and results in variation in device crosssections which affects the photonic/mechanical properties. These issues are mitigated by using metal based hard masks (such as $\mathrm{Al}$ ) which require additional pre-/post-processing steps. Other alternative approaches will require HSQ-like resists with better adhesion and stability to minimize the processing time, improve the device-to device reproducibility and up-scale the fabrication process.

The novel nanophotonic/nanomechanical devices realized via top-down methods might be affected by plasma induced damage. Furthermore, implantation based methods to create color centers create defects which degrade the properties of the final devices. These issues are inherent to top-down fabrication methods and need to be addressed by usage of alternative plasma mixtures, surface treatment methods, etc. [4]. Other methods to overcome this include combination of both bottom-up and top-down methods to realize diamond nano-structures with enhanced surface and optical properties [53].

In summary, while nanodevices in diamond have tremendously improved in recent years, large scale, low-cost availability of high-quality diamond, nano-structuring with minimum influence on device properties remain challenging to make these devices suitable for a broad range of applications in the fields of quantum information to sensing. Additionally, the realized devices should be easy to handle and integrate onto other photonic/mechanical platforms to develop multi-functional, on-chip hybrid systems.

Author Contributions: Writing—original draft preparation, D.R., O.R.O., E.N.; writing—review and editing, D.R., O.R.O., E.N.; funding acquisition, E.N. All authors have read and agreed to the published version of the manuscript.

Funding: This research was funded by the German Federal Ministry of Research grant number KKZ13N13547.

Conflicts of Interest: The authors declare no conflict of interest.

\section{References}

1. Castelletto, S.; Rosa, L.; Blackledge, J.; Al Abri, M.Z.; Boretti, A. Advances in diamond nanofabrication for ultrasensitive devices. Microsyst. Nanoeng. 2017, 3, 17061. [CrossRef] [PubMed]

2. Radtke, M.; Bernardi, E.; Slablab, A.; Nelz, R.; Neu, E. Nanoscale sensing based on nitrogen vacancy centers in single crystal diamond and nanodiamonds: Achievements and challenges. Nano Futur. 2019, 3, 042004. [CrossRef]

3. Nelz, R.; Görlitz, J.; Herrmann, D.; Slablab, A.; Challier, M.; Radtke, M.; Fischer, M.; Gsell, S.; Schreck, M.; Becher, C.; et al. Toward wafer-scale diamond nano- and quantum technologies. APL Mater. 2019, 7, 011108. [CrossRef]

4. Radtke, M.; Render, L.; Nelz, R.; Neu, E. Plasma treatments and photonic nanostructures for shallow nitrogen vacancy centers in diamond. Opt. Mater. Express 2019, 9, 4716-4733. [CrossRef]

5. Norman, V.A.; Majety, S.; Wang, Z.; Casey, W.H.; Curro, N.; Radulaski, M. Novel color center platforms enabling fundamental scientific discovery. InfoMat 2020, 1-24. [CrossRef]

6. Bradac, C.; Gao, W.; Forneris, J.; Trusheim, M.E.; Aharonovich, I. Quantum nanophotonics with group IV defects in diamond. Nat. Commun. 2019, 10, 5625. [CrossRef]

7. Bray, K.; Regan, B.; Trycz, A.; Previdi, R.; Seniutinas, G.; Ganesan, K.; Kianinia, M.; Kim, S.; Aharonovich, I. Single Crystal Diamond Membranes and Photonic Resonators Containing Germanium Vacancy Color Centers. ACS Photonics 2018, 5, 4817-4822. [CrossRef] 
8. Shang, N.; Papakonstantinou, P.; Wang, P.; Zakharov, A.; Palnitkar, U.; Lin, I.N.; Chu, M.; Stamboulis, A. Self-assembled growth, microstructure, and field-emission high-performance of ultrathin diamond nanorods. ACS Nano 2009, 3, 1032-1038. [CrossRef]

9. Hsu, C.H.; Cloutier, S.G.; Palefsky, S.; Xu, J. Synthesis of diamond nanowires using atmospheric-pressure chemical vapor deposition. Nano Lett. 2010, 10, 3272-3276. [CrossRef]

10. Malykhin, S.A.; Ismagilov, R.R.; Tuyakova, F.T.; Obraztsova, E.A.; Fedotov, P.V.; Ermakova, A.; Siyushev, P.; Katamadz, K.G.; Jelezko, F.; Rakovich, Y.P.; et al. Photoluminescent properties of single crystal diamond microneedles. Opt. Mater. 2018, 75, 49-55. [CrossRef]

11. Sotoma, S.; Terada, D.; Segawa, T.F.; Igarashi, R.; Harada, Y.; Shirakawa, M. Enrichment of ODMR-active nitrogen-vacancy centres in five-nanometre-sized detonation-synthesized nanodiamonds: Nanoprobes for temperature, angle and position. Sci. Rep. 2018, 8, 5463. [CrossRef] [PubMed]

12. Héritier, M.; Eichler, A.; Pan, Y.; Grob, U.; Shorubalko, I.; Krass, M.D.; Tao, Y.; Degen, C.L. Nanoladder Cantilevers Made from Diamond and Silicon. Nano Lett. 2018, 18, 1814-1818. [CrossRef] [PubMed]

13. Schröder, T.; Mouradian, S.L.; Zheng, J.; Trusheim, M.E.; Walsh, M.; Chen, E.H.; Li, L.; Bayn, I.; Englund, D. Quantum nanophotonics in diamond [Invited]. J. Opt. Soc. Am. B 2016, 33, B65-B83. [CrossRef]

14. Aharonovich, I.; Prawer, S. Fabrication strategies for diamond based ultra bright single photon sources. Diam. Relat. Mater. 2010, 19, 729-733. [CrossRef]

15. Element 6. Available online: https://www.e6.com/ (accessed on 6 December 2020).

16. Applied Diamond Inc. Available online: http://usapplieddiamond.com/ (accessed on 6 December 2020).

17. AuDiaTec. Available online: https://www.audiatec.de/en/ (accessed on 6 December 2020).

18. Pelliccione, M.; Jenkins, A.; Ovartchaiyapong, P.; Reetz, C.; Emmanouilidou, E.; Ni, N.; Jayich, A.C.B. Scanned probe imaging of nanoscale magnetism at cryogenic temperatures with a single-spin quantum sensor. Nat. Nanotechnol. 2016, 11, 700-705. [CrossRef] [PubMed]

19. Zhou, T.X.; Stöhr, R.J.; Yacoby, A. Scanning diamond NV center probes compatible with conventional AFM technology. Appl. Phys. Lett. 2017, 111, 163106. [CrossRef]

20. Hedrich, N.; Rohner, D.; Batzer, M.; Maletinsky, P.; Shields, B.J. Parabolic diamond scanning probes for single spin magnetic field imaging. Phys. Rev. Appl. 2020, 14, 064007. [CrossRef]

21. Bharadwaj, V.; Jedrkiewicz, O.; Hadden, J.P.; Sotillo, B.; Vázquez, M.R.; Dentella, P.; Fernandez, T.T.; Chiappini, A.; Giakoumaki, A.N.; Le Phu, T.; et al. Femtosecond laser written photonic and microfluidic circuits in diamond. J. Phys. Photonics 2019, 1, 22001. [CrossRef]

22. Eaton, S.M.; Hadden, J.P.; Bharadwaj, V.; Forneris, J.; Picollo, F.; Bosia, F.; Sotillo, B.; Giakoumaki, A.N.; Jedrkiewicz, O.; Chiappini, A.; et al. Quantum Micro-Nano Devices Fabricated in Diamond by Femtosecond Laser and Ion Irradiation. Adv. Quantum Technol. 2019, 2, 1900006. [CrossRef]

23. Ruf, M.; Ijspeert, M.; Van Dam, S.; De Jong, N.; Van Den Berg, H.; Evers, G.; Hanson, R. Optically Coherent Nitrogen-Vacancy Centers in Micrometer-Thin Etched Diamond Membranes. Nano Lett. 2019, 19, 3987-3992. [CrossRef]

24. Hensen, B.; Kalb, N.; Blok, M.S.; Dréau, A.E.; Reiserer, A.; Vermeulen, R.F.L.; Schouten, R.N.; Markham, M.; Twitchen, D.J.; Goodenough, K.; et al. Loophole-free Bell test using electron spins in diamond: Second experiment and additional analysis. Sci. Rep. 2016, 6, 30289. [CrossRef] [PubMed]

25. Choy, J.T.; Hausmann, B.J.M.; Burek, M.J.; Babinec, T.M.; Loncar, M. Nanofabrication of photonic devices from single- crystal diamond for quantum information processing (QIP). In Book Quantum Information Processing with Diamond: Principles and Applications; Prawer, S., Aharonovich, I., Eds.; Elsevier: Amsterdam, The Netherlands, 2014; pp. 98-123. [CrossRef]

26. Xu, L.; Yuan, H.; Zhang, N.; Zhang, J.; Bian, G.; Fan, P.; Li, M.; Zhang, C.; Zhai, Y.; Fang, J. High-efficiency fluorescence collection for NV-center ensembles in diamond. Opt. Express 2019, 27, 10787-10797. [CrossRef] [PubMed]

27. Piracha, A.H.; Ganesan, K.; Lau, D.W.M.; Stacey, A.; McGuinness, L.P.; Tomljenovic-Hanic, S.; Prawer, S. Scalable fabrication of high-quality, ultra-thin single crystal diamond membrane windows. Nanoscale 2016, 8, 6860-6865. [CrossRef] [PubMed]

28. Cady, J.V.; Michel, O.; Lee, K.W.; Patel, R.N.; Sarabalis, C.J.; Safavi-Naeni, A.H.; Bleszynski Jayich, A.C. Diamond optomechanical crystals with embedded nitrogen-vacancy centers. Quant. Sci. Tech. 2019, 4, 024009. [CrossRef]

29. Becker, J.N.; Pingault, B.; Groß, D.; Gündoğan, M.; Kukharchyk, N.; Markham, M.; Edmonds, A.; Atatüre, M.; Bushev, P.; Becher, C. All-Optical Control of the Silicon-Vacancy Spin in Diamond at Millikelvin Temperatures. Phys. Rev. Lett. 2018, 120, 053603. [CrossRef]

30. Siyushev, P.; Metsch, M.H.; Ijaz, A.; Binder, J.M.; Bhaskar, M.K.; Sukachev, D.D.; Sipahigil, A.; Evans, R.E.; Nguyen, C.T.; Lukin, M.D.; et al. Optical and microwave control of germanium-vacancy center spins in diamond. Phys. Rev. B 2017, 8, 081201. [CrossRef]

31. Burek, M.J.; Meuwly, C.; Evans, R.E.; Bhaskar, M.K.; Sipahigil, A.; Meesala, S.; MacHielse, B.; Sukachev, D.D.; Nguyen, C.T.; Pacheco, J.L.; et al. Fiber-Coupled Diamond Quantum Nanophotonic Interface. Phys. Rev. Appl. 2017, 8, 0240260. [CrossRef]

32. Zhang, J.L.; Sun, S.; Burek, M.J.; Dory, C.; Tzeng, Y.K.; Fischer, K.A.; Kelaita, Y.; Lagoudakis, K.G.; Radulaski, M.; Shen, Z.-X.; et al. Strongly Cavity-Enhanced Spontaneous Emission from Silicon- Vacancy Centers in Diamond. Nano Lett. 2018, 18, 1360-1365. [CrossRef] 
33. Nguyen, C.T.; Sukachev, D.D.; Bhaskar, M.K.; MacHielse, B.; Levonian, D.S.; Knall, E.N.; Stroganov, P.; Chia, C.; Burek, M.J.; Riedinger, R.; et al. An integrated nanophotonic quantum register based on silicon-vacancy spins in diamond. Phys. Revi. B 2019, 100, 165428. [CrossRef]

34. Mouradian, S.; Wan, N.H.; Schroeder, T.; Englund, D. Rectangular photonic crystal nanobeam cavities in bulk diamond. Appl. Phys. Lett. 2017, 111, 021103. [CrossRef]

35. Wan, N.H.; Mouradian, S.; Englund, D. Two-dimensional photonic crystal slab nanocavities on bulk single-crystal diamond. Appl. Phys. Lett. 2018, 112, 141102. [CrossRef]

36. Zheng, J.; Lienhard, B.; Doerk, G.; Cotlet, M.; Bersin, E.; Kim, H.S.; Byun, Y.C.; Nam, C.Y.; Kim, J.; Black, C.T.; et al. Top-down fabrication of high- uniformity nanodiamonds by self-assembled block copolymer masks. Sci. Rep. 2019, 9, 6914. [CrossRef] [PubMed]

37. Li, L.; Bayn, I.; Lu, M.; Nam, C.-Y.; Schröder, T.; Stein, A.; Harris, N.C.; Englund, D. Nanofabrication on unconventional substrates using transferred hard masks. Sci. Rep. 2015, 5, 7802. [CrossRef] [PubMed]

38. Schreck, M.; Gsell, S.; Brescia, R.; Fischer, M. Ion bombardment induced buried lateral growth: The key mechanism for the synthesis of single crystal diamond wafers. Sci. Rep. 2017, 7, 44462. [CrossRef]

39. Machielse, B.; Bogdanovic, S.; Meesala, S.; Gauthier, S.; Burek, M.J.; Joe, G.; Chalupnik, M.; Sohn, Y.I.; Holzgrafe, J.; Evans, R.E.; et al. Quantum Interference of Electromechanically Stabilized Emitters in Nanophotonic Devices. Phys. Rev. X 2019, 9, 031022. [CrossRef]

40. Atikian, H.A.; Latawiec, P.; Burek, M.J.; Sohn, Y.I.; Meesala, S.; Gravel, N.; Kouki, A.B.; Lončar, M. Freestanding nanostructures via reactive ion beam angled etching. APL Photonics 2017, 2, 051301. [CrossRef]

41. Huang, T.; Grote, R.R.; Mann, S.A.; Hopper, D.A.; Exarhos, A.L.; Lopez, G.G.; Kaighn, G.R.; Garnett, E.C.; Bassett, L.C. A monolithic immersion metalens for imaging solid-state quantum emitters. Nat. Commun. 2019, 10, 2392. [CrossRef]

42. Wan, N.H.; Shields, B.J.; Kim, D.; Lienhard, B.; Walsh, M.; Bakhru, H.; Schroeder, T.; Englund, D. Efficient Extraction of Light from a Nitrogen-Vacancy Center in a Diamond Parabolic Reflector. Nano Lett. 2018, 18, 2787-2793. [CrossRef]

43. Häußler, S.; Benedikter, J.; Bray, K.; Regan, B.; Dietrich, A.; Twamley, J.; Aharonovich, I.; Hunger, D.; Kubanek, A. Diamond photonics platform based on silicon vacancy centers in a single-crystal diamond membrane and a fiber cavity. Phys. Rev. B 2019, 99, 165310. [CrossRef]

44. Trycz, A.; Regan, B.; Kianinia, M.; Bray, K.; Toth, M.; Aharonovich, I. Bottom up engineering of single crystal diamond membranes with germanium vacancy color centers. Opti. Mat. Express 2019, 9, 4708-4715. [CrossRef]

45. Khanaliloo, B.; Mitchell, M.; Hryciw, A.C.; Barclay, P.E. High-Q/V Monolithic Diamond Microdisks Fabricated with Quasiisotropic Etching. Nano. Lett. 2015, 15, 5131-5136. [CrossRef] [PubMed]

46. Atikian, H.; Latawiec, P.; Xiong, X.; Meesala, S.; Gauthier, S.; Wintz, D.; Randi, J.; Bernot, D.; DeFrances, S.; Thomas, J.; et al. Diamond Mirror for High Power Lasers. arXiv 2020, arXiv:1909.06458v1.

47. Hopper, D.A.; Shulevitz, H.J.; Bassett, L.C. Spin Readout Techniques of the Nitrogen-Vacancy Center in Diamond. Micromachines 2018, 9, 437. [CrossRef] [PubMed]

48. Babinec, T.M.; Hausmann, B.J.M.; Khan, M.; Zhang, Y.; Maze, J.R.; Hemmer, P.R.; Lončar, M. A diamond nanowire single-photon source. Nat. Nanotech. 2010, 5, 195-199. [CrossRef]

49. Neu, E.; Appel, P.; Ganzhorn, M.; Miguel-Sánchez, J.; Lesik, M.; Mille, V.; Jacques, V.; Tallaire, A.; Achard, J.; Maletinsky, P. Photonic nano-structures on (111)-oriented diamond. Appl. Phys. Lett. 2016, 104, 153108. [CrossRef]

50. Osterkamp, C.; Mango, M.; Lang, J.; Balasubramanian, P.; Teraji, T.; Naydenov, B.; Jelezko, F. Engineering preferentially-aligned nitrogen-vacancy centre ensembles in CVD grown diamond. Sci. Rep. 2019, 9, 5786. [CrossRef]

51. Regan, B.; Aghajamali, A.; Froech, J.; Tran, T.T.; Scott, J.; Bishop, J.; Suarez-Martinez, I.; Liu, Y.; Cairney, J.M.; Marks, N.A.; et al. Plastic Deformation of Single-Crystal Diamond Nanopillars. Adv. Mat. 2020, 32, 1906458. [CrossRef]

52. Xie, L.; Zhou, T.X.; Stöhr, R.J.; Yacoby, A. Crystallographic Orientation Dependent Reactive Ion Etching in Single Crystal Diamond. Adv. Mater. 2018, 30, 1705501. [CrossRef]

53. Batzer, M.; Shields, B.; Neu, E.; Widmann, C.; Giese, C.; Nebel, C.; Maletinsky, P. Single crystal diamond pyramids for applications in nanoscale quantum sensing. Opt. Mater. Express 2020, 10, 492-500. [CrossRef]

54. Marseglia, L.; Aha, K.S.; Joy, A.A.; Schröder, T.; Englund, D.; Jelezko, F.; Walsworth, R.; Pacheco, J.L.; Perry, D.L.; Bielejec, E.S.; et al. Bright nanowire single photon source based on SiV centers in diamond. Opt. Express 2018, 26, 80-89. [CrossRef]

55. Zhang, J.L.; Lagoudakis, K.G.; Tzeng, Y.K.; Dory, C.; Radulaski, M.; Kelaita, Y.; Fischer, K.A.; Sun, S.; Shen, Z.X.; Melosh, N.A.; et al. Complete coherent control of silicon vacancies in diamond nanopillars containing single defect centers. Optica 2017, 4, 1317-1321. [CrossRef]

56. Felgen, N.; Naydenov, B.; Jelezko, F.; Reithmaier, J.P.; Popov, C. Homoepitaxial Diamond Structures with Incorporated SiV Centers. Phys. Stat. Soli. A 2018, 215, 1800371. [CrossRef]

57. Rugar, A.E.; Dory, C.; Sun, S.; Vučković, J. Characterization of optical and spin properties of single tin-vacancy centers in diamond nanopillars. Phys. Rev. B 2019, 99, 205417. [CrossRef]

58. Jeon, S.W.; Lee, J.; Jung, H.; Han, S.W.; Cho, Y.W.; Kim, Y.S.; Lim, H.T.; Kim, Y.; Niethammer, M.; Lim, W.C.; et al. Bright Nitrogen-Vacancy Centers in Diamond Inverted Nanocones. ACS Photonics 2020, 7, 2739-2747. [CrossRef]

59. Radtke, M.; Nelz, R.; Slablab, A.; Neu, E. Reliable nanofabrication of single-crystal diamond photonic nanostructures for nanoscale sensing. Micromachines 2019, 10, 718. [CrossRef] 
60. Maletinsky, P.; Hong, S.; Grinolds, M.S.; Hausmann, B.; Lukin, M.D.; Walsworth, R.L.; Loncar, M.; Yacoby, A. A robust scanning diamond sensor for nanoscale imaging with single nitrogen-vacancy centres. Nat. Nanotech. 2012, 7, 320-324. [CrossRef]

61. Häberle, T.; Schmid-Lorch, D.; Reinhard, F.; Wrachtrup, J. Nanoscale nuclear magnetic imaging with chemical contrast. Nat. Nanotech. 2015, 10, 125-128. [CrossRef]

62. Van Der Sar, T.; Casola, F.; Walsworth, R.; Yacoby, A. Nanometre-scale probing of spin waves using single electron spins. Nat. Commun. 2015, 6, 7886. [CrossRef]

63. Ernst, S.; Irber, D.M.; Waeber, A.M.; Braunbeck, G.; Reinhard, F. A Planar Scanning Probe Microscope. ACS Photonics 2019, 6, 327-331. [CrossRef]

64. Giese, C.; Quellmalz, P.; Knittel, P. Development of All-Diamond Scanning Probes Based on Faraday Cage Angled Etching Techniques. MRS Adv. 2020, 5, 1899-1907. [CrossRef]

65. Appel, P.; Neu, E.; Ganzhorn, M.; Barfuss, A.; Batzer, M.; Gratz, M.; Tschöpe, A.; Maletinsky, P. Fabrication of all diamond scanning probes for nanoscale magnetometry. Rev. Sci. Instr. 2017, 87, 063703. [CrossRef] [PubMed]

66. Rohner, D.; Happacher, J.; Reiser, P.; Tschudin, M.A.; Tallaire, A.; Achard, J.; Shields, B.J.; Maletinsky, P. (111)-oriented, single crystal diamond tips for nanoscale scanning probe imaging of out-of-plane magnetic fields. Appl. Phys. Lett. 2019, 115, 192401. [CrossRef]

67. Steinert, S.; Dolde, F.; Neumann, P.; Aird, A.; Naydenov, B.; Balasubramanian, G.; Jelezko, F.; Wrachtrup, J. High sensitivity magnetic imaging using an array of spins in diamond. Rev. Sci. Instr. 2016, 81, 043705. [CrossRef] [PubMed]

68. Momenzadeh, S.A.; Stöhr, R.J.; de Oliveira, F.F.; Brunner, A.; Denisenko, A.; Yang, S.; Reinhard, F.; Wrachtrup, J. Nanoengineered Diamond Waveguide as a Robust Bright Platform for Nanomagnetometry Using Shallow Nitrogen Vacancy Centers. Nano Lett. 2015, 15, 165-169. [CrossRef] [PubMed]

69. Mclellan, C.A.; Myers, B.A.; Kraemer, S.; Ohno, K.; Awschalom, D.D.; Bleszynski Jayich, A.C. Patterned Formation of Highly Coherent Nitrogen-Vacancy Centers Using a Focused Electron Irradiation Technique. Nano Lett. 2016, 16, 2450-2454. [CrossRef]

70. De Oliveira, F.F.; Antonov, D.; Wang, Y.; Neumann, P.; Momenzadeh, S.A.; Häußermann, T.; Pasquarelli, A.; Denisenko, A.; Wrachtrup, J. Tailoring spin defects in diamond by lattice charging. Nat. Commun. 2017, 8, 15409. [CrossRef]

71. Lühmann, T.; John, R.; Wunderlich, R.; Meijer, J.; Pezzagna, S. Coulomb-driven single defect engineering for scalable qubits and spin sensors in diamond. Nat. Commun. 2019, 10, 4956. [CrossRef]

72. Sangtawesin, S.; Dwyer, B.L.; Srinivasan, S.; Allred, J.J.; Rodgers, L.V.H.; De Greve, K.; Stacey, A.; Dontschuk, N.; O’Donnell, K.M.; $\mathrm{Hu}$, D.; et al. Origins of Diamond Surface Noise Probed by Correlating Single-Spin Measurements with Surface Spectroscopy. Phys. Rev. X 2019, 9, 031052. [CrossRef]

73. Kawai, S.; Yamano, H.; Sonoda, T.; Kato, K.; Buendia, J.J.; Kageura, T.; Fukuda, R.; Okada, T.; Tanii, T.; Higuchi, T.; et al. Nitrogen-Terminated Diamond Surface for Nanoscale NMR by Shallow Nitrogen-Vacancy Centers. J. Phys. Chem. C 2019, 123, 3594-3604. [CrossRef]

74. Herbschleb, E.D.; Kato, H.; Maruyama, Y.; Danjo, T.; Makino, T.; Yamasaki, S.; Ohki, I.; Hayashi, K.; Morishita, H.; Fujiwara, M.; et al. Ultra-long coherence times amongst room-temperature solid-state spins. Nat. Commun. 2019, 10, 3766. [CrossRef]

75. Kleinlein, J.; Borzenko, T.; Münzhuber, F.; Brehm, J.; Kiessling, T.; Molenkamp, L.W. NV-center diamond cantilevers: Extending the range of available fabrication methods. Microelect. Eng. 2016, 159, 70-74. [CrossRef]

76. Rondin, L.; Tetienne, J.P.; Spinicelli, P.; Dal Savio, C.; Karrai, K.; Dantelle, G.; Thiaville, A.; Rohart, S.; Roch, J.F.; Jacques, V. Nanoscale magnetic field mapping with a single spin scanning probe magnetometer. Appl. Phys. Lett. 2012, 15, 153118. [CrossRef]

77. Tao, Y.; Boss, J.M.; Moores, B.A.; Degen, C.L. Single-crystal diamond nanomechanical resonators with quality factors exceeding one million. Nat. Commun. 2014, 5, 3638. [CrossRef] [PubMed]

78. Tao, Y.; Navaretti, P.; Hauert, R.; Grob, U.; Poggio, M.; Degen, C.L. Permanent reduction of dissipation in nanomechanical Si resonators by chemical surface protection. Nanotechnology 2015, 26, 465501. [CrossRef]

79. Maity, S.; Shao, L.; Sohn, Y.I.; Meesala, S.; Machielse, B.; Bielejec, E.; Markham, M.; Lončar, M. Spectral alignment of single-photon emitters in diamond using strain gradient. Phys. Rev. Appl. 2018, 10, 024050. [CrossRef]

80. Mitchell, M.; Lake, D.P.; Barclay, P.E. Optomechanically amplified wavelength conversion in diamond microcavities. Optica 2019, 6, 832-838. [CrossRef]

81. Kehayias, P.; Jarmola, A.; Mosavian, N.; Fescenko, I.; Benito, F.M.; Laraoui, A.; Smits, J.; Bougas, L.; Budker, D.; Neumann, A.; et al. Solution nuclear magnetic resonance spectroscopy on a nanostructured diamond chip. Nat. Commun. 2017, 8, 188. [CrossRef] 